\title{
Reduced anterior cingulate cortex volume induced by chronic stress correlates with increased behavioral emotionality and decreased synaptic puncta density
}

Keith A. Misquitta ${ }^{1,2}$, Amy Miles ${ }^{1}$, Thomas D. Prevot ${ }^{1,4}$, Jaime K. Knoch ${ }^{1,2}$, Corey Fee ${ }^{1,2}$,

Dwight F. Newton ${ }^{1,2}$, Jacob Ellegood ${ }^{3}$, Jason P. Lerch ${ }^{3,5,6}$, Etienne Sibille ${ }^{1,2,4}$, Yuliya S.

$$
\text { Nikolova }^{1,4} \text {, Mounira Banasr }{ }^{1,2,4^{*}}
$$

${ }^{1}$ Campbell Family Mental Health Research Institute, Centre for Addiction and Mental Health (CAMH), Toronto, Canada.

${ }^{2}$ Departments of Pharmacology and Toxicology, University of Toronto, Toronto, Canada.

${ }^{3}$ Mouse Imaging Centre (MICe), Hospital for Sick Children, Toronto, Canada.

${ }^{4}$ Department of Psychiatry, University of Toronto, Toronto, Canada

${ }^{5}$ Wellcome Centre for Integrative Neuroimaging, FMRIB, Nuffield Department of Clinical Neuroscience, The University of Oxford, Oxford, UK

${ }^{6}$ Department of Medical Biophyics, The University of Toronto, Toronto, Canada

Contributions: Chronic restraint stress and Behavioral testing (KAM, TDP, CF), MRI and analysis (KAM, JKK, JE, JPL, DFN), Structural covariance analysis (AM,YN), Immunohistochemistry (KAM), study design, interpretation of the results and writing of the manuscript (KAM, ES, MB).

\section{Figures: 5 Figures}

\section{Tables: None}

\section{Supplementary Materials: 1 File}

*Corresponding author:

Mounira Banasr, PhD,

CAMH, 250 College Street,

Toronto, ON, M5T1R8, Canada,

Tel: 416-557-8302

Email: mounira.banasr@camh.ca 


\begin{abstract}
Clinical and preclinical studies report that chronic stress induces behavioral deficits as well as volumetric and synaptic alterations in corticolimbic brain regions including the anterior cingulate cortex (ACC), amygdala (AMY), nucleus accumbens (NAc) and hippocampus (HPC). Here, we aimed to investigate the volumetric changes associated with chronic restraint stress (CRS) and link these changes to the CRS-induced behavioral and synaptic deficits. We first confirmed that CRS increases behavioral emotionality, defined as collective scoring of anxiety- and anhedonialike behaviors. We then demonstrated that CRS induced a reduction of total brain volume which negatively correlated with behavioral emotionality. Region-specific analysis identified that only the ACC showed significant decrease in volume following CRS ( $p<0.05)$. Reduced ACC correlated with increased behavioral emotionality $(\mathrm{r}=-0.56 ; \mathrm{p}=0.0003)$. Although not significantly altered by CRS, AMY and NAc (but not the HPC) volumes were negatively correlated with behavioral emotionality. Finally, using structural covariance network analysis to assess shared volumetric variances between the corticolimbic brain regions and associated structures, we found a progressive decreased ACC degree and increased AMY degree following CRS. At the cellular level, reduced ACC volume correlated with decreased PSD95 (but not VGLUT1) puncta density $(\mathrm{r}=0.35, \mathrm{p}<0.05)$, which also correlated with increased behavioral emotionality ( $\mathrm{r}=-0.44, \mathrm{p}<0.01$ ), suggesting that altered synaptic strength is an underlying substrate of CRS volumetric and behavioral effects. Our results demonstrate that CRS effects on ACC volume and synaptic density are linked to behavioral emotionality and highlight key ACC structural and morphological alterations relevant to stress-related illnesses including mood and anxiety disorders.
\end{abstract}

Key Words: Chronic stress, Anterior cingulate cortex, Corticolimbic circuit, Depression, Synaptic markers, MRI 


\section{Highlights:}

1) Chronic restraint stress (CRS) decreases anterior cingulate cortex (ACC) volume

2) ACC and amygdala (AMY) volumes negatively correlate with behavioral emotionality

3) CRS decreased the strength and degree of the ACC structural covariance network

4) CRS increased the strength and degree of the AMY structural covariance network

5) PSD95 puncta density correlates with behavioral emotionality and ACC volume. 


\section{Introduction}

Exposure to stressful environment triggers a variety of adaptive responses followed by the return to a homeostatic state. However, when stress exposure becomes chronic, the stress response gradually becomes maladaptive and results in detrimental effects (McEwen, 2017). Indeed, exposure to stressful situations can lead to emotional dysregulation (Hammen et al., 2009) and is a major risk factor in the development of psychiatric illnesses, including major depressive disorder (MDD) (Kessler, 1997) and anxiety disorders (Kessler et al., 2003). The mechanisms involved in the maladaptive response to chronic stress include functional dysregulation within the corticolimbic circuit (Hariri and Holmes, 2015). This circuit, which is highly conserved across species, encompasses the amygdala (AMY), hippocampus (HPC), nucleus accumbens (NAc) and regions of the prefrontal cortex (PFC) that include the anterior cingulate cortex (ACC). These four brain regions are key structures implicated in emotion regulation and functional dysregulations of and have all been implicated in MDD and anxiety disorders (Ai et al., 2015; Ge et al., 2019; Johnstone et al., 2007; Klauser et al., 2015; Rao et al., 2016; Rodríguez-Cano et al., 2014; Tang et al., 2013; Webb et al.; Ye et al., 2015; Zhao et al., 2014). This circuit is responsible for maintaining the homeostatic balance following stress exposure. The function of each brain region of the corticolimbic circuit is influenced by numerous efferent and afferent projections from other brain regions, collectively creating a network critical for a healthy stress response as well as in pathological deficits involved in stress-related illnesses, such as MDD (Kim et al., 2011; Kong et al., 2013).

Neuroimaging studies are valuable tools to identify macroscale structural and functional alterations associated with neuropsychiatric disorders. Numerous magnetic resonance imaging (MRI) studies have observed volumetric changes in corticolimbic brain regions as well as brainwide structural adaptations associated with MDD (McKinnon et al., 2009; Schmaal et al., 2017; Schmaal et al., 2020; Schmaal et al., 2016; Treadway et al., 2015). Particularly, MRI studies identified decreases in volume of cortical areas including the ACC (Hajek et al., 2008; Koolschijn et al., 2009b; Schmaal et al., 2017) and hippocampal regions in MDD patients (Campbell et al., 2004; Kaymak et al., 2010; Koolschijn et al., 2009a; Malykhin et al., 2010; McKinnon et al., 2009; Schmaal et al., 2016; Steffens et al., 2011; Videbech, 2004). Further studies have described progressive changes associated with cortical thinning and greater number of prior major depressive episodes (Macqueen et al., 2003; Treadway et al., 2015). However 
mixed results have been reported regarding structural alterations of the AMY and NAc. Increased, decreased or no changes in volume of the AMY have been described (Arnone et al., 2012; Frodl et al., 2003; Hamilton et al., 2008; Schmaal et al., 2016). Regarding the NAc, MRI structural findings are limited and inconsistent, where for example one study reported increased volume of this brain region associated with MDD (Abdallah et al., 2017), while another study has reported decreased NAc volume with lifetime MDD (Ancelin et al., 2019).

Postmortem studies attribute these volumetric changes identified using MRI to altered neuronal complexity and synaptic loss or gain in the PFC (Kang et al 2012) and the HPC (Boldrini et al., 2013; Cobb et al., 2016; Cobb et al., 2013; Stockmeier et al., 2004). More specifically, MDD is associated with decreases in size of neuron soma, dendritic architecture and synaptic loss in the PFC (Bianchi et al., 2005; Kang et al., 2012; Rajkowska et al., 1999). Gene and protein expression of synaptic markers were reported reduced in the PFC primarily focusing on the ACC (Scifo et al., 2018; Shukla et al., 2020) in the HPC (Duric et al., 2013), while increased in the NAc and the AMY (Labonté et al., 2017).

To better understand the pathophysiology of chronic stress-related disorders such as MDD, chronic stress-based animal models are used to investigate the biological mechanisms that may underlie changes resulting in the dysfunction of the corticolimbic system. Such models have been extensively investigated to assess depressive-like behaviours, (Banasr and Duman, 2007; Nikolova et al., 2018; Willner, 2017) antidepressant efficacy (Banasr and Duman, 2007; Prevot et al., 2019b) and to determine sex-specific pathophysiological mechanisms (Guilloux et al., 2011; Seney and Sibille, 2014). Synaptic and morphological changes are found in chronic stress models, e.g., dendritic atrophy and spine loss in regions of the PFC (Cook and Wellman, 2004; Eiland et al., 2012) and hippocampus (Cook and Wellman, 2004; Eiland et al., 2012; Hill et al., 2011; Li et al., 2011; Radley et al., 2004; Radley et al., 2005; Son et al., 2012; Vyas et al., 2002; Wellman, 2001) or hypertrophy in the AMY (Eiland et al., 2012; Hill et al., 2013; Vyas et al., 2006; Vyas et al., 2002). Most of these studies used the chronic restraint stress (CRS), which is the model we chose for the present study to establish links between synaptic, volumetric and behavioral alterations associated with CRS.

Recent advancements in rodent structural MRI have proven to be valuable in studying the morphological changes associated with chronic stress across experimental models (Anacker et al., 2016; Kassem et al., 2013; Lee et al., 2009; Magalhães et al., 2018; Nikolova et al., 2018). 
These studies allow for the simultaneous investigation of morphological changes in multiple brain regions and can thus facilitate the identification of whole-brain network adaptations to chronic stress. In support of this notion, we recently used MRI to link unpredictable chronic mild stress with volumetric and structural covariance network changes centered on the AMY with depressive-like behavior and altered synaptic marker density (Nikolova et al., 2018).

In the present study, we aimed to examine the effects of chronic stress at the structural level using MRI-measured volumes, focusing on key brain regions of corticolimbic circuit, the PFC, HPC, AMY and NAc. The role of these volumetric changes on behavior was investigated by identifying potential relationships between the chronic stress-induced volumetric changes in these brain regions and behavior (anxiety-, anhedonia-like and cognitive behavioral deficits). We then analyzed the consequences of chronic stress on structural covariance network patterns of these regions and sought to determine the underlying substrate of these changes by examining if regions with significant volumetric changes also showed altered synaptic density using immunohistochemistry. Finally, an unbiased exploratory whole brain volume analysis was performed in all animals to investigate the brain-wide structural changes associated with chronic stress exposure.

\section{$\underline{2 \text { Methods }}$}

\subsection{Animals}

Eight-week old C57BL/6 mice (Jackson Laboratory, Bar Harbor, Maine, USA) were single housed under normal conditions with a $12 \mathrm{hr}$ light/dark cycle and provided with ad libitum access to food and water. All animals were habituated to the facility prior for 1 week to the start of the experimental procedures. All procedures were performed in accordance with institutional and Canadian Council on Animal Care (CCAC) procedural and ethical guidelines.

\section{$2.2 C R S$}

Animals were placed for a 1 -hour period inside $50 \mathrm{~mL}$ Falcon ${ }^{\circledR}$ tubes with air holes cut into each end, twice daily (minimum $2 \mathrm{hrs}$ apart) for 2 or 5 weeks ( $\mathrm{n}=12$ per group). Control animals were handled daily (Prevot et al., 2019a; Prevot et al., 2019b). We opted for examining the effects of 2 and 5 weeks of CRS since these two time points are, on average, the shortest and longest most studied durations of CRS used the literature for determining the effects of chronic stress on 
synaptic and behavioral changes (Prevot et al., 2019b; Qiao et al., 2016). The 3 groups (50\% females) were tested in all behavioral tests by an experimenter blinded to animal's group assignment. In this study, physical changes and anhedonia-like behavior were assessed using classical parameters and tests used in chronic stress studies, such as the weight of the animals, the state of their coat and sucrose preference (Prevot et al., 2019b; Willner, 2017). We also assessed anxiety-like behaviors using the novelty suppressed feeding (NSF) and phenotyper tests since chronic stress induces consistent deficits in these tests across strains, sex and experiments as compared to less reliable anxiety tests such as in the elevated plus maze or open field tests (Prevot et al., 2019b).

\subsection{Coat State Quality and Weight Measurements}

Seven anatomical regions (head, neck, dorsal/ventral coat, forepaws, hindpaw and tail) on each mouse were assessed where each region was given a score of either $0,0.5$ or 1 from maintained (0) to unkempt (1). Values for each region were summed up into a single score for each animal. Weight was recorded at the same time as coat state quality to determine weight gain or loss (measured in grams) associated the CRS exposure (Nollet et al., 2013). Coat state assessment was performed weekly on day 7, 15, 21, 28, 35 (Figure 1A).

\subsection{Sucrose Consumption Test (SCT)}

Before the start of CRS, animals were habituated to a $1 \%$ sucrose solution (Sigma, MO, USA) for $48 \mathrm{hrs}$ and are then fluid deprived for 16 hours overnight. Sucrose consumption was measured the next morning for a 1-hour period. The same procedure was performed for water consumption after two days. For each follow-up SCT, animals were re-habituated to the solution (sucrose or water) for 24 hours, fluids were then removed for 16 hours and consumption was measured for a 1-hour test (Duric et al., 2017). This test was performed weekly on day 7, 15, 21, 28, 35 (Figure 1A).

\subsection{PhenoTyper Test (PT)}

The Noldus ${ }^{\mathrm{TM}}$ PhenoTyper (Leesburg VA, USA) is an observational home cage-like apparatus able to video track mice for extended periods of time (EthoVision 10 software). In the arena, there are two designated zones (food $[6.5 \times 15 \mathrm{~cm}]$ and shelter zones $[10 \times 10 \mathrm{~cm}])$ where amount of 
time spent was recorded during the animal's dark cycle (19:00-07:00). In addition, an anxiogenic spotlight challenge appearing over the food zone begins 4 hours into the dark cycle for a 1-hour period (23:00-24:00). Testing in the PhenoTyper box was performed weekly on days 0, 7, 14, 21, 28, 35 of the CRS exposure. From previous studies we identified that stress-exposed and control animals spend equivalent amounts of time avoiding the food zone in favor of the shelter during the spotlight challenge. However, animals subjected to chronic stress continue to avoid the food zone after the challenge (Prevot et al., 2019b). This behavior, which we defined as "residual avoidance" (RA), is highly specific of chronic stress exposure (Prevot et al., 2019b). RA in the shelter zone was measured as the difference between the time spent during the light challenge and the sum of the time spent avoiding the lit zone ( 5 hours post-challenge). Formula for RA for each mouse as followed:

$\left[\left(\Sigma\right.\right.$ Time $_{(12 \mathrm{am}-5 \mathrm{am})}-$ Time $\left._{(11 \mathrm{pm}-12 \mathrm{am})}\right) /$ Average control group $\left(\Sigma\right.$ Time $_{(12 \mathrm{am}-5 \mathrm{am})}-$ Time $\left._{(11 \mathrm{pm}-12 \mathrm{am})}\right)-$ 1] * 100, where "Time" is time spent in shelter zone.

The RA measure provides a percentage of avoidance the animal exhibits after the white light challenge normalized to the control group. A positive RA value indicates the animal's avoidance of the lit zone in favor of the shelter zone (Prevot et al., 2019b). This test was performed weekly the day after the sucrose preference test (Figure 1A).

\subsection{Novelty Suppressed Feeding (NSF)}

Mice are food deprived for 16 hours prior to testing. During testing, mice are placed into a novel arena $(45 \times 30 \times 27 \mathrm{~cm}$ ) with a single food pellet in the center under dim lit conditions (lux 2830). The latency to approach and feed on the pellet is measured (in seconds). Similar test is performed in the animal's home cage; home cage latency to feed was also measured to exclude experimental bias associated with appetite drive. This test was performed once at the end of the experiment on day 37 (Figure 1A).

\subsection{Y-maze}

The Y-maze is an apparatus with 3 arms $\left(26 \times 8 \times 13 \mathrm{~cm}, 120^{\circ}\right.$ apart $)$ and sliding doors. Mice are habituated to the apparatus and allowed to explore the maze containing distal cues for 10 mins for 2 consecutive days. Mice are then trained to alternate for 7 successive trials and each trial is separated by an inter-trial interval (ITI) period of 30s. Each trial begins with a 30 s period in the 
"start-box" after which the door is slid open allowing the animal to explore one side of the arm. The door to the arm the animals entered is immediately closed for a 30s period and choice of arm is recorded. Mice return to the "start-box" and similar consecutive trials proceed. The testing phase follows the same procedure as training phase, except that an ITI of 90s is used, and with the addition of a $8^{\text {th }}$ trial with a 5 s ITI to assess for potential motivational loss. In the event of a lack of alternation on that trial, animals are removed from the analysis. All animals alternated in the last trial in this study. Data is calculated as the percent mean alternation rate (number of alternation/number of trials $\times 100$ ). This is Y-maze spontaneous alternation task where the animals use spatial cues. No food reward was given. CRS animals were previously shown to display deficits in this working memory task (Prevot et al., 2019a). This test was performed once at the end of the experiment on day 39 (Figure 1A).

\subsection{Mouse Brain Tissue Preparation}

At the end of the experiment, mice were anesthetized with avertine $(125 \mathrm{mg} / \mathrm{kg}$, i.p.) and intracardially perfused using a Pharmacia minipump at a rate of approximately $100 \mathrm{~mL} / \mathrm{hr}$ with $30 \mathrm{ml}$ of 0.1M PBS containing 10U/mL heparin and 2mM ProHance (Bracco Diagnostics, NJ, USA), followed by $30 \mathrm{ml}$ of $4 \%$ paraformaldehyde solution containing $2 \mathrm{mM}$ ProHance (Cahill et al., 2012; Lerch et al., 2011). After trans-cardiac perfusion, mouse bodies were decapitated. Skin, muscles, lower jaw, ears, and the cartilaginous nose tip were removed. The skull containing the brain was placed in a post-fixative solution of $4 \%$ PFA $+2 \mathrm{mM}$ ProHance overnight at $4^{\circ} \mathrm{C}$. Samples were then transferred into a buffer storage solution of $0.1 \mathrm{M}$ PBS with $2 \mathrm{mM}$ ProHance and $0.02 \%$ sodium azide for a minimum of one month (De Guzman et al., 2016) using a 7.0 Tesla MRI scanner (Agilent Inc., Palo Alto, CA) as in Anacker et al. (2016).

\subsection{MRI Acquisition and Preprocessing}

Brain preparations and scanning was performed as described in Nikolova et al (2018). However, regional volume was parcellated into 182 brain regions excluding ventricles (instead of 159 as in Wheeler et al. (2015) and adapted from previous rodent MRI studies (Dorr et al., 2008; Richards et al., 2011; Steadman et al., 2014; Ullmann et al., 2013). To minimize multiple testing burden, all our MRI analyses focused on region of interest (ROI) volumes averaged across hemispheres. Analyses were conducted using deformation-based morphometry to determine the absolute brain 
volumes (in $\mathrm{mm}^{3}$ ). First, we conducted an initial a priori analysis focusing on the effects of chronic stress exposure on the volumes of 4 corticolimbic brain regions. This required summation of multiple subregions for the ACC (cingulate Area 24a, a', b, b'), and hippocampal formation (CA1, CA2, CA3 and dentate gyrus). None was necessary for the NAc and AMY. Second, we tested a follow-up a priori analysis focusing on 26 MDD associated brain regions as in (Nikolova et al., 2018). For consistency, all these adjusted brain regions were used in the first a priori analysis, Pearson correlations and structural covariance analysis. Third, we conducted a whole-brain unbiased analysis in which these regions were separated into 182 regions of interest (ROIs).

\subsection{Structural Covariance}

Using the 182 ROIs, correlational matrices were created separately for each group. Based on previous studies, all negative correlations were removed (Nikolova et al., 2018). All matrices were thresholded over a range of density thresholds (0.05-0.4) that identified the top 5-40\% strongest connections by increments of $1 \%$. All thresholded matrices were converted to weighted graphs. We tested pairwise group differences (control vs. CRS 2 weeks, CRS 2 weeks vs. CRS 5 weeks, control vs. CRS 5 weeks) in regional degree and degree rank across density thresholds from 0.05 to 0.40 in increments of 0.01 . Significance was determined using permutation testing $(n=10,000)$ and defined as $\geq 5$ consecutive density thresholds with $p \leq 0.05$. Analyses were performed for the ACC, HPC, NAc and AMY, and regional volumes were adjusted for sex and total brain volume (refer to Nikolova et al. (2018)). Cytoscape (v3.7.1, Systems Biology, Seattle, USA) was used to visualize these networks at the lowest density threshold where observed between-group effects were significant. Subnetwork visualization of ROIs with significant structural covariance network changes (ACC and AMY) was created using the Edge-Weighted Spring Embedded Layout option in Cytoscape, as in Kamada and Kawai (1989), where each node represents the volume of a particular ROI and each edge represents the strength of correlation in volume between interconnected nodes.

\subsection{Fluorescence Immunohistochemistry}

After ex-vivo MRI, brains were extracted from the skull and placed in a 30\% sucrose solution for cryoprotection. Free-floating sections ( $30 \mu \mathrm{m}$ thickness) were cut using a cryostat (Leica, Wezlar, 
German) and placed in a cryoprotectant solution (sucrose 30\%, polyvinyl-pyrrolidone-40 (1\%), $0.1 \mathrm{M}$ phosphate buffer (PB) and ethylene glycol $30 \%$ ) for storage at $-20^{\circ} \mathrm{C}$ until immunohistochemistry. Three free floating sections from the ACC (coordinates from Bregma 1.41 to $1.53 \mathrm{~mm}$ ) or the basolateral amygdala (BLA) (coordinates from Bregma -1.31 and -1.43 $\mathrm{mm}$ ) were used for the immunohistochemistry. The sections were selected to represent anterioposterior coordinates as similar as possible for each animal. Sections were rinsed in 1X PBS phosphate-buffered saline (PBS, 20min) at $4^{\circ} \mathrm{C}$ and incubated in a $0.01 \mathrm{M}$ sodium citrate in distilled $\mathrm{H}_{2} \mathrm{O}$ at $80^{\circ} \mathrm{C}$ for 15 minutes. Solution was allowed to cool to room temperature and sections placed in a $0.3 \% \mathrm{X}-100$-Triton solution for 20 minutes followed by 1 hour in $20 \%$ donkey serum in PBS. Sections were incubated overnight at $4{ }^{\circ} \mathrm{C}$ in PBS containing $2 \%$ donkey serum and primary antibodies for post-synaptic density protein 95 (PSD95; rabbit host, 1:100, Cell Signaling Technology, Danvers, MA, product \#2507, lot 2), and vesicular glutamate transporter 1 (VGLUT1; guinea pig host, 1:500, Synaptic Systems, Goettingen, Germany, product \#135304, lot 135304/31). Sections were rinsed in PBS and incubated at $4^{\circ} \mathrm{C}$ in $\mathrm{PBS}$ containing $2 \%$ donkey serum and secondary antibodies conjugated to anti-rabbit Cy3 (Jackson ImmunoResearch Inc., West Grove, PA) and anti-guinea pig CF405M (Biotium, Hayward, CA), which were used to detect PSD95, and VGLUT1 immunoreactivity, respectively (2 hours, donkey host, 1:500 for all). Sections were placed through a final wash in PBS for 20 mins. Sections were mounted onto Superfrost plus gold slides (Fisher Scientific, Massachusetts, United States), coverslipped (Vectashield Antifade Mounting Media, Vector Laboratories, Burlingame, $\mathrm{CA}$ ), and stored at $4{ }^{\circ} \mathrm{C}$ until imaging and puncta quantification analysis

\subsection{Confocal Microscopy and Puncta Analysis}

Confocal imaging of the ACC and BLA using an Olympus IX83 inverted microscope equipped with a spinning disk confocal unit and Hamamatsu Orca-Flash4.0 V2 digital CMOS camera using a 60X 1.4 NA SC oil immersion objective. SlideBook 6 (Intelligent Imaging Innovations, Denver, CO) was used to capture images. All images used for quantification were obtained from acquisition of approximately 40 stacks (covering $~ 4 \mu \mathrm{m}$ thickness) of top layer imaged. Image $\mathrm{z}$ stacks were taken with a step size of $0.1 \mu \mathrm{m}$ based on Nyquist rate to acquire the minimal optimal sampling density. Using stereological available tools in SlideBook 6, five image stacks $(50 \times 50 \times 4 \mu \mathrm{m})$ were randomly taken for each hemisphere of the ACC (focusing on cingulate area 
24) and the amygdala (focusing on the BLA). All images were taken using the same exposure settings for each channel and blind deconvolution was performed using Autoquant (Media Cybernetics, Inc., Rockville, MD). Puncta analysis was conducted using IMARIS v9.5.1 software (BitPlane, Badenerstrasse, Zurich, Switzerland) which compiles z-stacked images into a 3D rendering. Quality parameters, a threshold setting for the intensity of light at the center of each spot, was set to for optimal puncta density quantification for PSD95 (250 for ACC and 150 BLA) and for VGLUT1 (200 for ACC and 650 for BLA). A background subtraction with a maximum pixel diameter (xy-axis) of 0.35 and a point spread function width (z-axis) 0.5 was set. All results were calculated as the density in $\mu \mathrm{m}^{3}$.

\subsection{Statistical analyses}

Using SPSS statistical software (IBM, NY), analysis of variance (ANOVA), followed by Fisher's post-hoc analysis, was performed on all behavioral tests. Repeated measures ANOVA was performed on the PhenoTyper, sucrose consumption, weight and coat state quality longitudinal assessments. A z-score normalization was performed to reduce behavioral data into one score for emotionality behavior. This calculation identifies the standard deviation of each group with respect to the mean of the controls. Z-scores were calculated from data obtained on the last testing in the PhenoTyper (residual avoidance), sucrose consumption, and NSF latency to bite tests and were averaged to create a z-emotionality score (Guilloux et al., 2011). Our a priori hypotheses of stress-induced volume changes in the 4 corticolimbic brain regions were tested using SPSS and separate ANCOVAs, including ROI volume as the dependent variable and total brain volume (TBV) and sex as regressors, followed by Fisher's post hoc tests. Pearson correlation was used to assess relationships between z-emotionality and absolute volume change for the amygdala, ACC, NAc and hippocampus (with/without adjusting for TBV and sex). Similar analyses were performed with the 26 MDD-associated brain regions and with all 182 brain regions, and results were corrected for multiple comparisons using false discovery rate (FDR, q<0.05). Associations between ROI volumes, adjusted for TBV, and Z-emotionality scores were tested in $\mathrm{R}$ using Pearson correlation followed by FDR correction $(\mathrm{q}<0.05)$. Similarly, associations between TBV-adjusted ROI volumes and percent alternations in the Ymaze test were assessed using a multi-trial binary logistic regression $(\mathrm{p}<0.05)$. Finally, Pearson correlation was used to assess links between changes in synaptic puncta of PSD95 and VGLUT1 
and ROI volume and z-score emotionality in SPSS, while logistic regression was used to assess links between Y-maze percent alternation and puncta density in R.

\section{Results}

3.1 CRS increases z-emotionality and decreases Y-maze performances assessed at 2 and 5 weeks

Supplementary Material details results obtained with longitudinal assessments of behavioral changes in the PT (Supplementary Figure 1) as well as sucrose consumption, weight and coat state changes, performed weekly to monitor efficacy of CRS exposure throughout the experiment (Supplementary Figure 2). Differences between sexes were analyzed for all the tests (Supplementary Figures 3 and 4).

During the final week of testing, endpoint tests included the coat state assessment, NSF, PT and sucrose consumption to assess the effects of CRS on behavioral emotionality. In the NSF test, ANOVA of latency to bite revealed a significant main effect of stress $\left(\mathrm{F}_{(2,30)}=4.149 ; \mathrm{p}<\right.$ 0.05; Figure 1B). Post hoc analysis identified a significant increase in latency to bite between the animal group subjected to 5 weeks of CRS compared with the control mouse group $(\mathrm{p}<0.05)$. No significant main effect of sex and stress $\mathrm{x}$ sex interaction were found (Supplementary Figure 3). Appetite drive as measured by home-cage latency to feed revealed no significant differences between groups.

In the PT, RA in the shelter zone was calculated as a measure of anxiety behavior as in Prevot et al. (2019). ANOVA of shelter RA revealed a significant main effect of stress $\left(\mathrm{F}_{(2,30)}=\right.$ 23.788; $\mathrm{p}<0.0001)$, sex $\left(\mathrm{F}_{(1,30)}=37.526 ; \mathrm{p}<0.0001\right)$ and stress $\mathrm{x} \operatorname{sex}\left(\mathrm{F}_{(2,30)}=11.259 ; \mathrm{p}<\right.$ 0.001) explained by a significant increase in RA in mice exposed to both 2 and 5 weeks of CRS as compared to controls $(\mathrm{p}<0.05)$ (Figure 1C). We also identified a significant increase in RA in 2 and 5 weeks CRS exposed female mice as compared to female controls and in CRS male mice compared to male controls (Supplementary Figure 3). Further ANOVA revealed a greater RA in females compared to males in mice exposed to both 2 and 5 weeks of CRS (Supplementary Figure 3).

The sucrose consumption test was measured as an index of anhedonia-like behavior and was conducted weekly (Supplementary Figure 2). The last sucrose consumption test performed on week 5, revealed a significant main effect of stress $\left(\mathrm{F}_{(2,30)}=4.403 ; \mathrm{p}<0.05\right)$ and $\operatorname{sex}\left(\mathrm{F}_{(1,30)}=\right.$ 7.005; $\mathrm{p}<0.05)$ but no stress $\mathrm{x}$ sex interaction was found. Post hoc analysis identified a significant decrease in sucrose consumption in mice exposed to both 2 and 5 weeks of CRS 
compared to control mice ( $\mathrm{p}<0.05$; Figure 1D). Furthermore, a significant decrease in sucrose consumption in females exposed to 2 and 5 weeks of CRS as compared with control females $(\mathrm{p}<0.05)$ was found. This was not evident in males (Supplementary Figure 3).

The NSF, PT and sucrose consumption tests were z-scored and compiled into a zemotionality score. We found a significant main effect of stress $\left(F_{(2,30)}=12.344 ; p<0.0001\right)$, $\operatorname{sex}\left(\mathrm{F}_{(1,30)}=17.604 ; \mathrm{p}<0.0001\right)$ and stress $\mathrm{x}$ sex interaction $\left(\mathrm{F}_{(2,30)}=4.449 ; \mathrm{p}<0.05\right)$. Post hoc analysis identified a significant increase in z-emotionality score in mice exposed to 2 and 5 weeks of CRS compared to control mice ( $\mathrm{p}<0.05$; Figure 1F). Increased z-emotionality score was significant in female mice exposed to 2 and 5 weeks of CRS compared to males exposed or not to CRS ( $<<0.05$; Figure 2A ). Further, males exposed to 5 weeks of CRS revealed a significant increase in $\mathrm{z}$-emotionality as compared to the male no stress group $(\mathrm{p}<0.05$; Figure 2A).

Lastly the Y-maze test was used to assess effects of CRS on cognition, specifically working memory. We found a significant effect of stress $\left(\mathrm{F}_{(2,30)}=10.524 ; \mathrm{p}<0.001\right)$ and sex $\left(\mathrm{F}_{(1,30)}=5.909 ; \mathrm{p}<0.05\right)$ on percent alternations, but no stress $\mathrm{x}$ sex interaction. Post hoc analysis revealed a significant decrease in percent alternations in mice exposed to 2 or 5 weeks of CRS as compared to controls ( $p<0.05$; Figure 1G); this effect was significant only in males (Supplementary Figure 3). Analysis of coats state quality at 5 weeks showed a significant main effect of stress $\left(\mathrm{F}_{(2,33)}=5.797 ; \mathrm{p}<0.01\right)$. Post hoc analysis revealed a significant increase in coat state score between mice exposed to 5 weeks of CRS as compared to controls and 2 weeks CRS exposed mice $(\mathrm{p}<0.05 ;$ Figure 1E).

\subsection{Total brain volume decreases in CRS-exposed mice and correlates with z-emotionality}

Total brain volume significantly decreased $\left(\mathrm{F}_{(2,30)}=5.666 ; \mathrm{p}<0.001\right)$ in animals exposed to 2 or 5 weeks of CRS as compared to controls. There was no main effect of sex, however we found a stress $x$ sex interaction $\left(\mathrm{F}_{(2,30)}=4.298 ; \mathrm{p}<0.05\right)$ revealing that only females showed a significant decrease in TBV when exposed to 2 or 5 weeks of CRS ( $\mathrm{p}<0.05$, for both CRS group relative to control group, Figure 2B). Pearson's correlation revealed a significant negative correlation between TBV and z-emotionality score across groups $(r=-0.495 ; p=0.002$, Figure 2C). While such correlation was detected in females $(r=-0.608 ; p=0.007$, Figure 2C), no significant 
correlation was found in male mice $(r=-0.336$; $p=0.173$, Figure 2C). Using binary logistic regression, we found that TBV did not predict $\mathrm{Y}$-maze performance $(\mathrm{p}>0.05)$.

3.3 ACC volume decreases in CRS-exposed mice and corticolimbic limbic regional volumes negatively correlate with z-emotionality.

The initial a priori analysis using ANCOVA revealed significant PFC volume changes following $\operatorname{CRS}\left(\mathrm{F}_{(2,31)}=3.826 ; \mathrm{p}<0.05\right)$ using TBV as a covariate and sex as a cofactor; however, this effect was primarily driven to a significant effect of stress on the $A C C$ volume $\left(F_{(2,31)}=4.053 ; p<0.05\right.$; Figure 3A) as no other PFC regions showed significant changes. Post hoc analysis revealed a significant decrease in ACC volume in mice exposed to 2 or 5 weeks of CRS, compared to control mice $(\mathrm{p}<0.05)$. However, no significant changes in volume were found in the AMY, NAc and HPC following CRS (Figure 3). The absolute volumes of the ACC $(r=-0.56 ; \mathrm{p}=0.0003$; Figure 3B), AMY ( $r=-0.35 ; \mathrm{p}=0.03$; Figure 3F) and NAc $(r=-0.41 ; \mathrm{p}=0.01$; Figure 3H) negatively correlated with z-emotionality score. The HPC volume did not correlate with zemotionality score. This analysis was performed using absolute volume of each brain region and did not survive when TBV was included in the analysis as a covariate and sex as a cofactor ( $\mathrm{p}>0.05)$. Using binary logistic regression, we found that volume of the ACC, AMY, NAc and HPC did not predict Y-maze performances (Supplementary Table 3).

3.4 CRS alters volumes of 2 of the 26 ROIs associated with MDD and 9 negatively correlated with z-emotionality

A follow-up analysis was performed across 26 ROIs involved in MDD (Supplementary Table 1) selected as in Nikolova et al. (2018). ANCOVA analysis using TBV as covariate and sex as cofactor revealed that the cingulate cortex area $24 \mathrm{~B}$ and the striatum showed significant reductions in volume in the 2 or 5 weeks CRS groups as compared to controls after FDR correction for multiple comparisons $(q<0.05)$. Pearson correlations were performed on the 26 ROI volumes corrected for TBV revealed a significant negative correlation between $\mathrm{z}$ emotionality and volume of the cingulate cortex area 24A, 24B, hypothalamus, insular region, medial parietal association cortex, midbrain, NAc, striatum and thalamus $(\mathrm{q}<0.05$, Supplementary Table 2). Logistic regression analysis was then conducted to determine association between 26 ROIs and Y-maze performance and revealed significant links with 
cingulate cortex area 29c, temporal association area and thalamus $(\mathrm{p}<0.05$, Supplementary Table 3); however, these changes did not survive FDR correction. A follow-up whole-brain regional volume analysis of 182 brain regions was performed in all animals where only the primary somatosensory cortex: trunk region volume was significantly reduced in the 2 weeks CRS group compared to controls $(\mathrm{q}<0.05)$. The same analysis performed only in females showed that none of the 182 ROI display significant volumetric changes following CRS (all q>0.05) (Supplementary Table 4 and 5).

\subsection{Progressive decrease in structural covariance network (SCN) degree in ACC and increase in} SCN degree in the Amygdala in CRS exposed animals.

SCN analysis can identify synchronous structural changes based on mutual trophic effects or shared plasticity between regions and networks (Mechelli, 2005). Additional recent work suggests structural covariance patterns further correspond to inter-regional structural connectivity and transcriptomic similarity (Yee et al., 2018). For the four selected corticolimbic brain regions, degree centrality was assessed to identify their position and "hubness", respective to brain wide SCN changes. We found no significant group differences in regional degree or degree rank when comparing (1) the control group vs. 2 weeks CRS group or (2) the 2 weeks CRS group vs. 5 weeks CRS group. When comparing the control group and the 5 weeks CRS group, we found no significant group differences in degree or degree rank of the HPC or NAc (data not shown). However, comparison between the control and 5 weeks CRS groups for the ACC revealed that degree was significantly lower at multiple consecutive density thresholds in the 5 weeks CRS group compared to controls (density $=0.13-0.19, \mathrm{p} \leq 0.05$, Figure 4A), with group differences largest at density $=0.16(\mathrm{p}=0.012)$. Correspondingly, ACC degree rank was significantly lower in mice subjected to 5 weeks CRS compared to controls (density $=0.12$ $0.18, \mathrm{p} \leq 0.05)$. Using the first significant density threshold, the ACC SCN neighboring nodes (density $=0.12$ ) are represented in Figure 4B for all three groups (see Supplementary Table 6 for additional details). When comparing the control group and the 5 weeks CRS group, we found significant group differences in AMY degree whereby degree was significantly greater in the 5 weeks CRS group than in the control group at several consecutive density thresholds (density = $0.08-0.12, p \leq 0.05$, Figure 4C). Group differences were largest at density $=0.08(p=0.001)$. Amygdala degree rank was significantly lower in the 5 weeks CRS group than in the control 
group at several consecutive density thresholds (density $=0.05-0.10, \mathrm{p} \leq 0.05$ ). Structural covariance networks for the AMY are represented in Figure 4D, where they are visualized at the first of the five aforementioned consecutive significant density thresholds (density= 0.08). Supplementary Table 7 indicates neighboring nodes, the volume of which covaries with that of the AMY at the chosen density threshold. Connected and unconnected nodes are indicated with 1 and 0, respectively. For the AMY, we confirmed increased SCN degree and degree rank in animals subjected to chronic stress (Nikolova et al., 2018).

\subsection{PSD95 puncta density in the ACC correlates with ACC volume and z-emotionality}

Given the significant effect of CRS exposure on the ACC volume, we investigated the changes in density of marker expression of pre- and post-synaptic compartments, PSD95 (Figure 5A-C) and VGLUT1 (Supplementary Figure 6), respectively. Quantitative analysis of PSD95 puncta density using analytic IMARIS quantitative spot counting software revealed a near-trend toward a main effect of stress $\left(\mathrm{F}_{(2,28)}=2.408 ; \mathrm{p}=0.11\right)$, significant main effect of $\operatorname{sex}\left(\mathrm{F}_{(1,28)}=6.462\right.$; $\mathrm{p}<0.05$ ) and no stress $\mathrm{x}$ sex interaction (Figure 5D). Overall females displayed lower PSD95 puncta density but no significant differences in density of puncta between groups was found (data not shown). Interestingly, we found a significant positive correlation between PSD95 puncta density with volume changes of the ACC $(r=0.35 ; \mathrm{p}=0.044$; Figure 5E). In addition, PSD95 puncta density was negatively correlated with z-emotionality score $(r=-0.439 ; \mathrm{p}=0.009$; Figure 5F). Similar analysis conducted on VGLUT1 puncta density in ACC revealed no significant effect of stress, sex or stress $\mathrm{x}$ sex interaction, and no correlation with either $\mathrm{z}$ emotionality or volume change of the ACC (Supplementary Figure 6). Using binary logistic regression, we found that PSD95 or VGLUT1 puncta densities did not predict Y-maze performances $\left(\chi^{2}=1.62\right.$ and $\chi^{2}=-0.578$, respectively). Given the significant effect of CRS exposure on SCN AMY, we also investigated the changes in PSD95 and VGLUT1 puncta density in the BLA and found no significant effects of stress or sex on PSD95 puncta density in this area. PSD95 puncta density in the BLA did not correlate with z-emotionality or AMY volume. However, CRS exposure induced an increase in VGLUT1 puncta density ( $<<0.05$; Supplementary Figure 7) which correlated with z-emotionality $(r=0.362, \mathrm{p}<0.05)$ but not with AMY volume (Supplementary Figure 7). 


\section{Discussion:}

The goal of this study was to use high resolution structural neuroimaging in rodents for studying brain-network adaptations underlying the behavioral deficits associated with chronic stress exposure. We showed that mice exposed to CRS displayed a progressive increase in anxiety-like behavior in the NSF and PhenoTyper tests, anhedonia-like behavior in the sucrose consumption test and impairment in working memory in the Y-maze test. These results confirmed that CRS results in increased behavioral emotionality and cognitive dysfunctions. Using ex-vivo MRI, we were able to identify an overall decrease in TBV (driven by reduced TBV in female mice) exposed to CRS. Correlation analyses revealed that reduced TBV was associated with increase in behavioral emotionality across sexes. Based on the literature in both animal and human studies of chronic stress and MDD, the ACC, AMY, NAc, and HPC were selected for our a priori analysis to identify changes in volume of corticolimbic structures. This analysis identified that CRS induced a significant decrease in volume of the ACC - a region of the mPFC commonly affected by chronic stress. Although no significant changes were observed in the other a priori corticolimbic brain regions, we were able to identify a link between the ACC, AMY and NAc volumes and behavioral emotionality deficits associated with CRS. We also confirmed that CRS-exposed mice show an increase in structural covariance degree of the AMY coupled with a decrease in ACC degree following 5 weeks of CRS exposure. The volumetric changes observed in the ACC were associated with PSD95 but not VGLUT1 puncta density. Additionally, reduced PSD95 puncta density in the ACC was negatively correlated with increased behavioral emotionality and positively correlated with reduced volumetric changes of this brain region. Finally, although CRS induced impairment in the Y-maze, performances in this test were not predicted by volumetric changes in specific brain region or synaptic puncta density in ACC. Altogether, chronic stress induced ACC volume reduction which was associated with reduced ACC structural covariance degree, PSD95 puncta density and increased behavioral emotionality, suggesting that altered synaptic strength is an underlying substrate of the volumetric and behavioral effects of CRS. Our results highlight a vulnerability of the ACC to chronic stress exposure and further implicate the ACC morphological alterations in stress-related illness including mood and anxiety disorders.

In accordance with the literature, our study confirmed that CRS induced behavioral emotionality in tests measuring anxiety- (Guilloux et al., 2011; Prevot et al., 2019b) and 
anhedonia-like behaviors (Banasr et al., 2010) as well as deficits in working memory (Prevot et al., 2019a). Prior work has shown that the effects of chronic stress are heterogeneous across sexes (Guilloux et al., 2011; Seney and Sibille, 2014). Here, we confirmed that female mice showed a progressive and higher magnitude of vulnerability to CRS exposure on anxiety- and anhedonia-like readouts compared to males (Guilloux et al., 2011). This is in accordance with human studies reporting greater prevalence of stress-induced symptoms of anxiety and comorbidity with anxiety disorders in women (Curry et al., 2014; Maeng and Milad, 2015; Schuch et al., 2014). These data critically emphasize the importance of using both sexes in experimental designs. Interestingly, in the Y-maze, a working memory task, deficits were found in male CRS mice. These results are in consensus with the literature describing deficits in the Ymaze appearing 7-10 days following CRS exposure (Prevot et al., 2019a). This result suggests that compared to male mice, females may exhibit lower susceptibility to cognitive impairments following chronic stress exposure. This hypothesis needs to be explicitly confirmed in future work but it is consistent with previous reports using other chronic stress rodent models (Conrad et al., 2003; Darcet et al., 2016). The progressive effects of chronic stress exposure were also confirmed on physical readouts such as degradation of coat state quality and lack of weight gain (Nikolova et al., 2018; Prevot et al., 2019b). Overall, regardless of sex differences our results identify behavioral emotionality deficits and cognitive impairments due to chronic stress exposure.

We also identified sex-specific patterns of volumetric changes, which complement prior rodent in vivo and ex-vivo MRI studies performed in male-only cohorts (Anacker et al., 2016; Lee et al., 2009; Magalhães et al., 2018; Nikolova et al., 2018). Specifically, we found that CRS induced an overall decrease in TBV driven by the female groups. Although, one prior study has reported TBV reductions in male rats following exposure to chronic unpredictable stress (Magalhães et al., 2018), other studies in male rodents have not reported such an effect (Lee et al., 2009; Nikolova et al., 2018). Imaging studies have associated between-group differences in TBV with variations in weight (O'Brien et al., 2011). In our study we found that although weight was significantly reduced by CRS, the association between weight and TBV did not account for much shared variance (data not shown); thus, weight was not regressed out in our analysis. The lack of consistency across studies regarding chronic stress-induced TBV changes in males may be due to the use of various chronic stress models, length of stress exposure or 
species (mouse or rat). Nevertheless, the female-specific reduction in TBV may also reflect greater susceptibility to chronic stress in female mice that may result in an overall brain atrophy. Studies have reported that intra-cranial volume was a more accurate readout than TBV to correct for, when studying conditions associated with neuronal atrophy (O'Brien et al., 2011). Interestingly, TBV reductions correlated with behavioral emotionality in both males and females. This suggests that a smaller TBV resulting from chronic stress might be an indicator of behavioral emotionality deficits. In human literature, total gray matter and TBV changes in MDD patients have been found but considered insignificant when compared to the general population (Zhuo et al., 2019). This overall brain reduction in rodents subjected to chronic stress may be the result of neuronal atrophy affecting numerous brain regions and being greater in females.

A priori analysis focusing on 4 corticolimbic brain regions identified the ACC as a brain region whose volume significantly decreased with CRS. We found a significant $\sim 3 \%$ decrease in ACC volume in mice exposed to either 2 or 5 weeks CRS. This confirms previous MRI findings describing decreased ACC volume in C57B1/6 mice following CRS (Kassem et al., 2013). In this latter study, The authors report a $16 \%$ reduction of ACC volume following 21 days of CRS (1 session of $6 \mathrm{~h}$ restraint per day) (Kassem et al., 2013). The difference in magnitude of change may be due to greater length of each CRS session (Kassem et al., 2013). From human MRI literature, ACC volume reductions have been identified in major depressive (Bora et al., 2012; Van Tol et al., 2010), anxiety (Van Tol et al., 2010), and panic disorders (Asami et al., 2008). This reduced volume was identified in MDD patients who have experienced multiple episodes (Bora et al., 2012), lifetime occurrence of MDD (Ancelin et al., 2019), and are seen in treatment non-responders (Liu et al., 2017). Regarding the HPC, contrary to previous studies describing decreased volume following CRS (Kassem et al., 2013; Lee et al., 2009), we found no HPC volume change. This may be due to differences in length or duration of the CRS procedures or the fact that we did not subdivide the dorsal hippocampus from the ventral hippocampus, the latter known to be more involved in emotion regulation and more susceptible to stress (Kheirbek and Hen, 2011). We also found no significant changes in absolute volumes of the AMY and NAc volume in CRS mice, confirming most rodent chronic stress MRI studies (Anacker et al., 2016; Kassem et al., 2013; Lee et al., 2009; Magalhães et al., 2018). However, we previously described a $\sim 3 \%$ increase in AMY relative volume following unpredictable chronic mild stress in BalbC 
male mice (Nikolova et al., 2018). Such inconsistencies in rodent chronic stress models mirror heterogeneous findings reporting variable results in human MDD patients with some studies describing larger, smaller or no changes in amygdala volume. In fact, smaller AMY volume has been attributed to greater number of episodes (Frodl et al., 2002; Frodl et al., 2003; Hamilton et al., 2008). Overall, in our study, we see volumetric changes in corticolimbic regions that may reflect a later state of the chronic stress response as they are somewhat similar to the volumetric changes reported with multiple episodes of MDD. Despite not finding significant changes in absolute volumes of corticolimbic brain regions (except for the ACC), we did identify strong correlations between volume of the AMY and NAc, and behavioral emotionality. Extending this first a priori analysis to MDD-associated brain regions allowed us to uncover additional brain regions of relevance to the expression of behavior emotionality deficits including multiple medial prefrontal subfields and key subcortical brain regions involved in sensory and emotion processing (thalamus, hypothalamus and midbrain). These correlations further emphasize the importance these regions have on the expression of depressive-like behavior and their role in the modulation of chronic stress response.

The seed-based analysis of structural covariance performed for the 4 corticolimbic brain regions identified that the $\mathrm{ACC}$ and the amygdala SCN connectivity patterns are affected by chronic stress. Specifically, we have identified a progressive CRS-induced decrease in the number of brain regions that structurally covaries with the ACC (i.e. the SCN degree of the ACC). Interestingly, this CRS-induced decrease in ACC degree is associated with a loss of connectivity with critical brain regions such as the NAc, medial amygdala, thalamus, bed nucleus of the stria terminalis (BNST) and multiple cortical subregions following 5 weeks CRS exposure. Human imaging studies showed that the medial prefrontal cortical network, including the medial orbital-frontal cortex, thalamus and limbic basal ganglia, is important in the processing of emotionally salient information (Price and Drevets, 2010). A reduced SCN degree could reflect a disconnect of the ACC of its network which may be responsible for, or at the very least linked with, elevated behavioral emotionality deficits found following CRS. Despite no significant AMY volumetric changes, we confirmed an increase in AMY SCN degree and increased synaptic puncta density with greater chronic stress exposure (Nikolova et al., 2018). Similar increase in AMY SCN degree was also found in a human cohort with history of childhood trauma (Nikolova et al., 2018). Our results suggest that the AMY SCN changes are conserved 
across stress models, sexes and strains as well as across species. The ACC and AMY SCN reorganization patterns suggest a decrease in ACC SCN connectivity coupled with an AMY SCN strengthening which maps onto synaptic puncta density in the ACC and increased density in the BLA respectively. Interestingly synaptic changes in these brain regions parallels SCN reorganization changes that may collectively be involved in the impaired emotional regulation associated with chronic exposure to stress.

One of the most common factors associated with brain volume changes is synaptic and dendritic reorganization. Here, we found a marginal decrease in PSD95 and no change in VGLUT1 puncta density in the ACC in CRS exposed mice. Decreases in PSD95 protein in the ACC have been identified in rodents subjected to various chronic stress models (Kang et al., 2012; Li et al., 2011; Qiao et al., 2016; Son et al., 2012). In the present study, PSD95 puncta density in the ACC correlated with volumetric change in the ACC, i.e., decreased volume is associated with decreases in the density of PSD95 puncta. Regarding VGLUT1 we found no significant changes in puncta density in the ACC. Human and rodent studies have identified decreases in mRNA expression of VGLUT1 (Uezato et al., 2009; Zink et al., 2010), analysis of protein expression following chronic stress exposure in the cortical brain regions have identified mixed results (Farley et al., 2012; Garcia-Garcia et al., 2009; Yu et al., 2018). Changes in VGLUT1 puncta did not correlate with volume changes in the ACC in this study. This lack of difference in ACC VGLUT1 expression in chronic stress mice compared to controls may be due to the fact that VGLUT1 protein can originate from other brain regions that project to the PFC unaffected by CRS (Collins et al., 2018; McGarry and Carter, 2017). Unlike VGLUT1, expression of PSD95 protein is postsynaptic, involved in synaptic strength and expressed by neurons endogenous to ACC which could explain why PSD95 puncta density was significantly correlated with the ACC volume changes. Since PSD95 puncta density changes may index altered synaptic density and strength (Meyer et al., 2014), our results suggest that these synaptic dysfunctions are underlying substrates for the reduced ACC volume and decreased ACC SCN associated with CRS, together responsible for behavioral emotionality deficits.

This study has several limitations. First, while we were able to detect some sexspecificity in the behavioral and structural changes induced by CRS, our study was not originally designed to examine sex differences and was underpowered to specifically investigate volumetric changes within each sex. A challenge in this study was that most of the CRS effects 
did not survive correction for TBV because of the unexpectedly reduced TBV in CRS. The large shared variance between TBV and a majority of brain regions and the CRS-induced TBV impacted our ability to detect region-specific changes due to CRS when TBV was added as a covariate. Perhaps controlling for intracranial volume when neuronal atrophy is expected could address this limitation, as with some imaging studies focusing on neurodegenerative disorders (O'Brien et al., 2011). Second, high resolution ex-vivo MRI can delineate relatively small brain structures (182 in this study) which can be beneficial to understand the involvement of certain sub-structures but adds difficulty to the analysis where very few changes are able to survive FDR correction. Although it might be beneficial in some rodent MRI studies to have a very precise atlas that can detect minute volumetric changes to small brain regions, such parcellation may impede the comparison of our findings to human MDD studies. Indeed, in many human MRI studies regional brain volume structures are not subdivided as extensively as in rodents and having a harmonized atlas between human and rodent MRI studies could bridge this gap. Nonetheless, we present results from whole-brain volumetric analyses including all regions in this refined parcellation scheme in order to foster discovery science and hypothesis generation for future studies. Third, in this study we were unable to establish a link between cognitive impairment and morphological (structural or synaptic) changes. This is perhaps due to the use of a single behavioral test assessing one cognitive dimension (working memory); further studies employing a more comprehensive behavioral assessment of cognition are needed to address this specific question. Despite these limitations, we were able to demonstrate that ACC volumetric changes induced by chronic stress correlated with behavioral changes and PSD95 puncta density, linking these macroscale and microscale alterations to behavioral deficits. Altogether, our results highlight a vulnerability of the ACC to chronic stress at multiple levels (volume, connectivity and spine) and its role in the expression of emotionality in behaviors relevant to stress-related illness including mood and anxiety disorders.

\section{Acknowledgements}

This work was supported by the Canadian Institute of Heath research (CIHR Project Grant PJT\#165852, PI: MB), the CAMH Discovery Fund (MB) and the Campbell Family Mental Health Research Institute of CAMH (MB, ES). YSN is supported by a Koerner New Scientist Award administered by the CAMH Foundation and an NSERC Discovery Grant (RGPIN-2020- 
07131). AM is supported by a CAMH Discovery Fund Postdoctoral Fellowship. JPL and JE are supported by the Ontario Brain Institute and CIHR.

\section{Conflicts of Interests}

The authors declare that they have no conflict of interest related with this work.

\section{References}

Abdallah, C. G., Jackowski, A., Salas, R., Gupta, S., Sato, J. R., Mao, X., Coplan, J. D., Shungu, D. C., Mathew, S. J., 2017. The Nucleus Accumbens and Ketamine Treatment in Major Depressive Disorder. Neuropsychopharmacology 42, 1739-1746.

Ai, H., Opmeer, E., Veltman, D., Wee, N. v. d., Buchem, M. v., Aleman, A., Tol, M. v., 2015. Brain Activation During Emotional Memory Processing Associated with Subsequent Course of Depression. Neuropsychopharmacology 40, 2454-2463.

Anacker, C., Scholz, J., O’Donnell, K. J., Allemang-Grand, R., Diorio, J., Bagot, R. C., Nestler, E. J., Hen, R., Lerch, J. P., Meaney, M. J., 2016. Neuroanatomic differences associated with stress susceptibility and resilience. Biological Psychiatry 79, 840-849.

Ancelin, M.-L., Carrière, I., Artero, S., Maller, J., Meslin, C., Ritchie, K., Ryan, J., Chaudieu, I., 2019. Lifetime major depression and grey-matter volume. Journal of Psychiatry \& Neuroscience 44, 45-53.

Arnone, D., McIntosh, A., Ebmeier, K., Munafò, M., Anderson, I., 2012. Magnetic resonance imaging studies in unipolar depression: systematic review and meta-regression analyses. European Neuropsychopharmacology 22, 1-16.

Asami, T., Hayano, F., Nakamura, M., Yamasue, H., Uehara, K., Otsuka, T., Roppongi, T., Nihashi, N., Inoue, T., Hirayasu, Y., 2008. Anterior cingulate cortex volume reduction in patients with panic disorder. Psychiatry and Clinical Neurosciences 62, 322-330.

Banasr, Chowdhury, G. M. I., Terwilliger, R., Newton, S. S., Duman, R. S., Behar, K. L., Sanacora, G., 2010. Glial pathology in an animal model of depression: reversal of stress-induced cellular, metabolic and behavioral deficits by the glutamate-modulating drug riluzole. Molecular psychiatry 15, 501-511.

Banasr, M., Duman, R. S., 2007. Regulation of neurogenesis and gliogenesis by stress and antidepressant treatment. CNS \& Neurological Disorders-Drug Targets (Formerly Current Drug Targets-CNS \& Neurological Disorders) 6, 311-320.

Bianchi, M., Hagan, J., Heidbreder, C., 2005. Neuronal plasticity, stress and depression: involvement of the cytoskeletal microtubular system? Current Drug Targets-CNS \& Neurological Disorders 4, 597-611.

Boldrini, M., Santiago, A. N., Hen, R., Dwork, A. J., Rosoklija, G. B., Tamir, H., Arango, V., John Mann, J., 2013. Hippocampal Granule Neuron Number and Dentate Gyrus Volume in 
Antidepressant-Treated and Untreated Major Depression. Neuropsychopharmacology 38, 10681077.

Bora, E., Fornito, A., Pantelis, C., Yücel, M., 2012. Gray matter abnormalities in major depressive disorder: a meta-analysis of voxel based morphometry studies. Journal of Affective Disorders 138, 9-18.

Cahill, L. S., Laliberté, C. L., Ellegood, J., Spring, S., Gleave, J. A., Eede, M. C., Lerch, J. P., Henkelman, R. M., 2012. Preparation of fixed mouse brains for MRI. Neuroimage 60, 933-939. Campbell, S., Marriott, M., Nahmias, C., Macqueen, G. M., 2004. Lower Hippocampal Volume in Patients Suffering From Depression: A Meta-Analysis. American Journal of Psychiatry 161, 598-607.

Cobb, J. A., O’Neill, K., Milner, J., Mahajan, G. J., Lawrence, T. J., May, W. L., MiguelHidalgo, J., Rajkowska, G., Stockmeier, C. A., 2016. Density of GFAP-immunoreactive astrocytes is decreased in left hippocampi in major depressive disorder. Neuroscience 316, 209220.

Cobb, J. A., Simpson, J., Mahajan, G. J., Overholser, J. C., Jurjus, G. J., Dieter, L., Herbst, N., May, W., Rajkowska, G., Stockmeier, C. A., 2013. Hippocampal volume and total cell numbers in major depressive disorder 47, 299-306.

Collins, D. P., Anastasiades, P. G., Marlin, J. J., Carter, A. G., 2018. Reciprocal Circuits Linking the Prefrontal Cortex with Dorsal and Ventral Thalamic Nuclei. Neuron 98, 366-379.e364.

Conrad, C. D., Grote, K. A., Hobbs, R. J., Ferayorni, A., 2003. Sex differences in spatial and non-spatial Y-maze performance after chronic stress. Neurobiology of learning and memory 79, 32-40.

Cook, S. C., Wellman, C. L., 2004. Chronic stress alters dendritic morphology in rat medial prefrontal cortex. Journal of neurobiology 60, 236-248.

Curry, J. F., Aubuchon-Endsley, N., Brancu, M., Runnals, J. J., Workgroup, R., Workgroup, V. M.-A. M. R., Fairbank, J. A., 2014. Lifetime major depression and comorbid disorders among current-era women veterans. Journal of Affective Disorders 152, 434-440.

Darcet, F., Gardier, A., Gaillard, R., David, D., Guilloux, J.-P., 2016. Cognitive Dysfunction in Major Depressive Disorder. A Translational Review in Animal Models of the Disease.

Pharmaceuticals 9, 9.

De Guzman, A. E., Wong, M. D., Gleave, J. A., Nieman, B. J., 2016. Variations in postperfusion immersion fixation and storage alter MRI measurements of mouse brain morphometry 142, 687-695.

Dorr, A., Lerch, J. P., Spring, S., Kabani, N., Henkelman, R. M., 2008. High resolution threedimensional brain atlas using an average magnetic resonance image of 40 adult $\mathrm{C} 57 \mathrm{Bl} / 6 \mathrm{~J}$ mice. Neuroimage 42, 60-69.

Duric, V., Banasr, M., Franklin, T., Lepack, A., Adham, N., Kiss, B., Gyertyán, I., Duman, R. S., 2017. Cariprazine Exhibits Anxiolytic and Dopamine D3 Receptor-Dependent Antidepressant Effects in the Chronic Stress Model. International Journal of Neuropsychopharmacology 20, 788-796.

Duric, V., Banasr, M., Stockmeier, C. A., Simen, A. A., Newton, S. S., Overholser, J. C., Jurjus, G. J., Dieter, L., Duman, R. S., 2013. Altered expression of synapse and glutamate related genes in post-mortem hippocampus of depressed subjects. International Journal of Neuropsychopharmacology 16, 69-82. 
Eiland, L., Ramroop, J., Hill, M. N., Manley, J., McEwen, B. S., 2012. Chronic juvenile stress produces corticolimbic dendritic architectural remodeling and modulates emotional behavior in male and female rats. Psychoneuroendocrinology 37, 39-47.

Farley, S., Dumas, S., El Mestikawy, S., Giros, B., 2012. Increased expression of the Vesicular Glutamate Transporter-1 (VGLUT1) in the prefrontal cortex correlates with differential vulnerability to chronic stress in various mouse strains: effects of fluoxetine and MK-801.

Neuropharmacology 62, 503-517.

Frodl, T., Meisenzahl, E., Zetzsche, T., Bottlender, R., Born, C., Groll, C., Jäger, M., Leinsinger, G., Hahn, K., Möller, H.-J., 2002. Enlargement of the amygdala in patients with a first episode of major depression. Biological Psychiatry 51, 708-714.

Frodl, T., Meisenzahl, E. M., Zetzsche, T., Born, C., Jäger, M., Groll, C., Bottlender, R., Leinsinger, G., Möller, H.-J., 2003. Larger amygdala volumes in first depressive episode as compared to recurrent major depression and healthy control subjects. Biological Psychiatry 53, 338-344.

Garcia-Garcia, A. L., Elizalde, N., Matrov, D., Harro, J., Wojcik, S. M., Venzala, E., Ramírez, M. J., Del Rio, J., Tordera, R. M., 2009. Increased Vulnerability to Depressive-Like Behavior of Mice with Decreased Expression of VGLUT1. Biological Psychiatry 66, 275-282.

Ge, R., Torres, I., Brown, J., Gregory, E., McLellan, E., Downar, J., Blumberge, D., Daskalakis, Z., Lam, R., Vila-Rodriguez, F., 2019. Functional disconnectivity of the hippocampal network and neural correlates of memory impairment in treatment-resistant depression. J Affect Disord 253, 248-256.

Guilloux, J.-P., Seney, M., Edgar, N., Sibille, E., 2011. Integrated behavioral z-scoring increases the sensitivity and reliability of behavioral phenotyping in mice: Relevance to emotionality and sex. Journal of Neuroscience Methods 197, 21-31.

Hajek, T., Kozeny, J., Kopecek, M., Alda, M., Höschl, C., 2008. Reduced subgenual cingulate volumes in mood disorders: a meta-analysis. Journal of psychiatry \& neuroscience: JPN 33, 91. Hamilton, J. P., Siemer, M., Gotlib, I. H., 2008. Amygdala volume in major depressive disorder: a meta-analysis of magnetic resonance imaging studies. Molecular psychiatry 13, 993.

Hammen, C., Kim, E. Y., Eberhart, N. K., Brennan, P. A., 2009. Chronic and acute stress and the prediction of major depression in women. Depression and Anxiety 26, 718-723.

Hariri, A. R., Holmes, A., 2015. Finding translation in stress research. Nature Neuroscience 18, 1347-1352.

Hill, M. N., Hillard, C. J., McEwen, B. S., 2011. Alterations in corticolimbic dendritic morphology and emotional behavior in cannabinoid CB1 receptor-deficient mice parallel the effects of chronic stress. Cerebral cortex 21, 2056-2064.

Hill, M. N., Kumar, S. A., Filipski, S. B., Iverson, M., Stuhr, K. L., Keith, J. M., Cravatt, B. F., Hillard, C. J., Chattarji, S., McEwen, B. S., 2013. Disruption of fatty acid amide hydrolase activity prevents the effects of chronic stress on anxiety and amygdalar microstructure.

Molecular psychiatry 18, 1125.

Johnstone, T., Reekum, C. v., Urry, H., Kalin, N., Davidson, R., 2007. Failure to regulate: counterproductive recruitment of top-down prefrontal-subcortical circuitry in major depression. $\mathrm{J}$ Neurosci 27, 8877-8884.

Kamada, T., Kawai, S., 1989. An algorithm for drawing general undirected graphs. Information processing letters 31, 7-15.

Kang, H. J., Voleti, B., Hajszan, T., Rajkowska, G., Stockmeier, C. A., Licznerski, P., Lepack, A., Majik, M. S., Jeong, L. S., Banasr, M., Son, H., Duman, R. S., 2012. Decreased expression of 
synapse-related genes and loss of synapses in major depressive disorder. Nature Medicine 18, 1413-1417.

Kassem, M. S., Lagopoulos, J., Stait-Gardner, T., Price, W. S., Chohan, T. W., Arnold, J. C., Hatton, S. N., Bennett, M. R., 2013. Stress-Induced Grey Matter Loss Determined by MRI Is Primarily Due to Loss of Dendrites and Their Synapses. Molecular Neurobiology 47, 645-661. Kaymak, S. U., Demir, B., Şentürk, S., Tatar, I., Aldur, M. M., Uluğ, B., 2010. Hippocampus, glucocorticoids and neurocognitive functions in patients with first-episode major depressive disorders. European Archives of Psychiatry and Clinical Neuroscience 260, 217-223.

Kessler, R. C., 1997. The effects of stressful life events on depression. Annual Review of Psychology 48, 191-214.

Kessler, R. C., Berglund, P., Demler, O., Jin, R., Koretz, D., Merikangas, K. R., Rush, A. J., Walters, E. E., Wang, P. S., 2003. The epidemiology of major depressive disorder: results from the National Comorbidity Survey Replication (NCS-R). JAMA 289, 3095-3105.

Kheirbek, M. A., Hen, R., 2011. Dorsal vs Ventral Hippocampal Neurogenesis: Implications for Cognition and Mood. Neuropsychopharmacology 36, 373-374.

Kim, M. J., Loucks, R. A., Palmer, A. L., Brown, A. C., Solomon, K. M., Marchante, A. N., Whalen, P. J., 2011. The structural and functional connectivity of the amygdala: From normal emotion to pathological anxiety. Behavioural Brain Research 223, 403-410.

Klauser, P., Fornito, A., Lorenzetti, V., Davey, C., Dwyer, D., Allen, N., Yücel, M., 2015. Cortico-limbic network abnormalities in individuals with current and past major depressive disorder. J Affect Disord 173, 45-52.

Kong, L., Chen, K., Womer, F., Jiang, W., Luo, X., Driesen, N., Liu, J., Blumberg, H., Tang, Y., $\mathrm{Xu}, \mathrm{K}$., Wang, F., 2013. Sex differences of gray matter morphology in cortico-limbic-striatal neural system in major depressive disorder. Journal of Psychiatric Research 47, 733-739. Koolschijn, P. C. M., van Haren, N. E., Lensvelt $\square$ Mulders, G. J., Hulshoff Pol, H. E., Kahn, R. S., 2009a. Brain volume abnormalities in major depressive disorder: A meta $\square$ analysis of magnetic resonance imaging studies. Human Brain Mapping 30, 3719-3735.

Koolschijn, P. C. M. P., Van Haren, N. E. M., Lensvelt-Mulders, G. J. L. M., Hulshoff Pol, H. E., Kahn, R. S., 2009b. Brain volume abnormalities in major depressive disorder: A metaanalysis of magnetic resonance imaging studies. Human Brain Mapping 30, 3719-3735.

Labonté, B., Engmann, O., Purushothaman, I., Menard, C., Wang, J., Tan, C., Scarpa, J. R., Moy, G., Loh, Y.-H. E., Cahill, M., Lorsch, Z. S., Hamilton, P. J., Calipari, E. S., Hodes, G. E., Issler, O., Kronman, H., Pfau, M., Obradovic, A. L. J., Dong, Y., Neve, R. L., Russo, S., Kasarskis, A., Tamminga, C., Mechawar, N., Turecki, G., Zhang, B., Shen, L., Nestler, E. J., 2017. Sex-specific transcriptional signatures in human depression. Nature Medicine 23, 1102-1111.

Lee, T., Jarome, T., Li, S.-J., Kim, J. J., Helmstetter, F. J., 2009. Chronic stress selectively reduces hippocampal volume in rats: a longitudinal magnetic resonance imaging study. NeuroReport 20, 1554-1558.

Lerch, J. P., Sled, J. G., Henkelman, R. M., 2011. MRI phenotyping of genetically altered mice. Methods Mol Biol 711, 349-361.

Li, N., Liu, R.-J., Dwyer, J. M., Banasr, M., Lee, B., Son, H., Li, X.-Y., Aghajanian, G., Duman, R. S., 2011. Glutamate N-methyl-D-aspartate receptor antagonists rapidly reverse behavioral and synaptic deficits caused by chronic stress exposure. Biological Psychiatry 69, 754-761. Liu, J., Xu, X., Luo, Q., Luo, Y., Chen, Y., Lui, S., Wu, M., Zhu, H., Kemp, G. J., Gong, Q., 2017. Brain grey matter volume alterations associated with antidepressant response in major depressive disorder. Scientific Reports 7. 
Macqueen, G. M., Campbell, S., McEwen, B. S., Macdonald, K., Amano, S., Joffe, R. T., Nahmias, C., Young, L. T., 2003. Course of illness, hippocampal function, and hippocampal volume in major depression. Proceedings of the National Academy of Sciences 100, 1387-1392. Maeng, L. Y., Milad, M. R., 2015. Sex differences in anxiety disorders: Interactions between fear, stress, and gonadal hormones. Hormones and Behavior 76, 106-117.

Magalhães, R., Barrière, D. A., Novais, A., Marques, F., Marques, P., Cerqueira, J., Sousa, J. C., Cachia, A., Boumezbeur, F., Bottlaender, M., Jay, T. M., Mériaux, S., Sousa, N., 2018. The dynamics of stress: a longitudinal MRI study of rat brain structure and connectome. Molecular psychiatry 23, 1998-2006.

Malykhin, N. V., Carter, R., Seres, P., Coupland, N. J., 2010. Structural changes in the hippocampus in major depressive disorder: contributions of disease and treatment. Journal of psychiatry \& neuroscience: JPN 35, 337.

McEwen, B. S., 2017. Neurobiological and Systemic Effects of Chronic Stress. Chronic Stress 1, 247054701769232.

McGarry, L. M., Carter, A. G., 2017. Prefrontal Cortex Drives Distinct Projection Neurons in the Basolateral Amygdala. Cell Reports 21, 1426-1433.

McKinnon, M. C., Yucel, K., Nazarov, A., MacQueen, G. M., 2009. A meta-analysis examining clinical predictors of hippocampal volume in patients with major depressive disorder. Journal of psychiatry \& neuroscience: JPN 34, 41.

Mechelli, A., 2005. Structural Covariance in the Human Cortex. Journal of Neuroscience 25, 8303-8310.

Meyer, D., Bonhoeffer, T., Scheuss, V., 2014. Balance and stability of synaptic structures during synaptic plasticity. Neuron $82,430-443$.

Nikolova, Y. S., Misquitta, K. A., Rocco, B. R., Prevot, T. D., Knodt, A. R., Ellegood, J., Voineskos, A. N., Lerch, J. P., Hariri, A. R., Sibille, E., Banasr, M., 2018. Shifting priorities: highly conserved behavioral and brain network adaptations to chronic stress across species. Translational Psychiatry 8.

Nollet, M., Guisquet, A. M. L., Belzung, C., 2013. Models of depression: unpredictable chronic mild stress in mice. Current protocols in pharmacology 61, 5.65. 61-65.65. 17.

O'Brien, L. M., Ziegler, D. A., Deutsch, C. K., Frazier, J. A., Herbert, M. R., Locascio, J. J., 2011. Statistical adjustments for brain size in volumetric neuroimaging studies: Some practical implications in methods. Psychiatry Research: Neuroimaging 193, 113-122.

Prevot, T. D., Li, G., Vidojevic, A., Misquitta, K. A., Fee, C., Santrac, A., Daniel, Michael, Kodali, R., Nicolas, Leggy, Scholze, P., Janet, Bojan, Banasr, M., James, Savic, M., Sibille, E., 2019a. Novel Benzodiazepine-Like Ligands with Various Anxiolytic, Antidepressant, or ProCognitive Profiles. Molecular Neuropsychiatry 5, 84-97.

Prevot, T. D., Misquitta, K. A., Fee, C., Newton, D. F., Chatterjee, D., Nikolova, Y. S., Sibille, E., Banasr, M., 2019b. Residual avoidance: A new, consistent and repeatable readout of chronic stress-induced conflict anxiety reversible by antidepressant treatment. Neuropharmacology 153 , 98-110.

Price, J. L., Drevets, W. C., 2010. Neurocircuitry of Mood Disorders.

Neuropsychopharmacology 35, 192-216.

Qiao, H., Li, M.-X., Xu, C., Chen, H.-B., An, S.-C., Ma, X.-M., 2016. Dendritic Spines in Depression: What We Learned from Animal Models. Neural Plasticity 2016, 1-26. 
Radley, J., Sisti, H., Hao, J., Rocher, A. B., McCall, T., Hof, P., McEwen, B., Morrison, J., 2004. Chronic behavioral stress induces apical dendritic reorganization in pyramidal neurons of the medial prefrontal cortex. Neuroscience 125, 1-6.

Radley, J. J., Rocher, A. B., Miller, M., Janssen, W. G., Liston, C., Hof, P. R., McEwen, B. S., Morrison, J. H., 2005. Repeated stress induces dendritic spine loss in the rat medial prefrontal cortex. Cerebral cortex 16, 313-320.

Rajkowska, G., Miguel-Hidalgo, J. J., Wei, J., Dilley, G., Pittman, S. D., Meltzer, H. Y., Overholser, J. C., Roth, B. L., Stockmeier, C. A., 1999. Morphometric evidence for neuronal and glial prefrontal cell pathology in major depression. Biological Psychiatry 45, 1085-1098.

Rao, J., Jenkins, L., Hymen, E., Feigon, M., Weisenbach, S., Zubieta, J., Langenecker, S., 2016. Differential Resting State Connectivity Patterns and Impaired Semantically Cued List Learning Test Performance in Early Course Remitted Major Depressive Disorder. J Int Neuropsychol Soc 22, 225-239.

Richards, K., Watson, C., Buckley, R. F., Kurniawan, N. D., Yang, Z., Keller, M. D., Beare, R., Bartlett, P. F., Egan, G. F., Galloway, G. J., 2011. Segmentation of the mouse hippocampal formation in magnetic resonance images. Neuroimage 58, 732-740.

Rodríguez-Cano, E., Sarró, S., Monté, G., Maristany, T., Salvador, R., McKenna, P., PomarolClotet, E., 2014. Evidence for structural and functional abnormality in the subgenual anterior cingulate cortex in major depressive disorder. Psychol Med 44, 3263-3273.

Schmaal, L., Hibar, D. P., Sämann, P. G., Hall, G. B., Baune, B. T., Jahanshad, N., Cheung, J. W., van Erp, T. G. M., Bos, D., Ikram, M. A., Vernooij, M. W., Niessen, W. J., Tiemeier, H., Hofman, A., Wittfeld, K., Grabe, H. J., Janowitz, D., Bülow, R., Selonke, M., Völzke, H., Grotegerd, D., Dannlowski, U., Arolt, V., Opel, N., Heindel, W., Kugel, H., Hoehn, D., Czisch, M., Couvy-Duchesne, B., Rentería, M. E., Strike, L. T., Wright, M. J., Mills, N. T., de Zubicaray, G. I., McMahon, K. L., Medland, S. E., Martin, N. G., Gillespie, N. A., GoyaMaldonado, R., Gruber, O., Krämer, B., Hatton, S. N., Lagopoulos, J., Hickie, I. B., Frodl, T., Carballedo, A., Frey, E. M., van Velzen, L. S., Penninx, B., van Tol, M. J., van der Wee, N. J., Davey, C. G., Harrison, B. J., Mwangi, B., Cao, B., Soares, J. C., Veer, I. M., Walter, H., Schoepf, D., Zurowski, B., Konrad, C., Schramm, E., Normann, C., Schnell, K., Sacchet, M. D., Gotlib, I. H., MacQueen, G. M., Godlewska, B. R., Nickson, T., McIntosh, A. M., Papmeyer, M., Whalley, H. C., Hall, J., Sussmann, J. E., Li, M., Walter, M., Aftanas, L., Brack, I., Bokhan, N. A., Thompson, P. M., Veltman, D. J., 2017. Cortical abnormalities in adults and adolescents with major depression based on brain scans from 20 cohorts worldwide in the ENIGMA Major Depressive Disorder Working Group. Mol Psychiatry 22, 900-909.

Schmaal, L., Pozzi, E., T, C. H., van Velzen, L. S., Veer, I. M., Opel, N., Van Someren, E. J. W., Han, L. K. M., Aftanas, L., Aleman, A., Baune, B. T., Berger, K., Blanken, T. F., Capitão, L., Couvy-Duchesne, B., K, R. C., Dannlowski, U., Davey, C., Erwin-Grabner, T., Evans, J., Frodl, T., Fu, C. H. Y., Godlewska, B., Gotlib, I. H., Goya-Maldonado, R., Grabe, H. J., Groenewold, N. A., Grotegerd, D., Gruber, O., Gutman, B. A., Hall, G. B., Harrison, B. J., Hatton, S. N., Hermesdorf, M., Hickie, I. B., Hilland, E., Irungu, B., Jonassen, R., Kelly, S., Kircher, T., Klimes-Dougan, B., Krug, A., Landrø, N. I., Lagopoulos, J., Leerssen, J., Li, M., Linden, D. E. J., MacMaster, F. P., A, M. M., Mehler, D. M. A., Nenadić, I., Penninx, B., Portella, M. J., Reneman, L., Rentería, M. E., Sacchet, M. D., P, G. S., Schrantee, A., Sim, K., Soares, J. C., Stein, D. J., Tozzi, L., van Der Wee, N. J. A., van Tol, M. J., Vermeiren, R., Vives-Gilabert, Y., Walter, H., Walter, M., Whalley, H. C., Wittfeld, K., Whittle, S., Wright, M. J., Yang, T. T., Zarate, C., Jr., Thomopoulos, S. I., Jahanshad, N., Thompson, P. M., Veltman, D. J., 2020. 
ENIGMA MDD: seven years of global neuroimaging studies of major depression through worldwide data sharing. Transl Psychiatry 10, 172.

Schmaal, L., Veltman, D. J., van Erp, T. G., Sämann, P. G., Frodl, T., Jahanshad, N., Loehrer, E., Tiemeier, H., Hofman, A., Niessen, W. J., Vernooij, M. W., Ikram, M. A., Wittfeld, K., Grabe, H. J., Block, A., Hegenscheid, K., Völzke, H., Hoehn, D., Czisch, M., Lagopoulos, J., Hatton, S. N., Hickie, I. B., Goya-Maldonado, R., Krämer, B., Gruber, O., Couvy-Duchesne, B., Rentería, M. E., Strike, L. T., Mills, N. T., de Zubicaray, G. I., McMahon, K. L., Medland, S. E., Martin, N. G., Gillespie, N. A., Wright, M. J., Hall, G. B., MacQueen, G. M., Frey, E. M., Carballedo, A., van Velzen, L. S., van Tol, M. J., van der Wee, N. J., Veer, I. M., Walter, H., Schnell, K., Schramm, E., Normann, C., Schoepf, D., Konrad, C., Zurowski, B., Nickson, T., McIntosh, A. M., Papmeyer, M., Whalley, H. C., Sussmann, J. E., Godlewska, B. R., Cowen, P. J., Fischer, F. H., Rose, M., Penninx, B. W., Thompson, P. M., Hibar, D. P., 2016. Subcortical brain alterations in major depressive disorder: findings from the ENIGMA Major Depressive Disorder working group. Mol Psychiatry 21, 806-812.

Schuch, J. J. J., Roest, A. M., Nolen, W. A., Penninx, B. W. J. H., de Jonge, P., 2014. Gender differences in major depressive disorder: results from the Netherlands study of depression and anxiety. Journal of Affective Disorders 156, 156-163.

Scifo, E., Pabba, M., Kapadia, F., Ma, T., Lewis, D. A., Tseng, G. C., Sibille, E., 2018. Sustained Molecular Pathology Across Episodes and Remission in Major Depressive Disorder. Biological Psychiatry 83, 81-89.

Seney, M. L., Sibille, E., 2014. Sex differences in mood disorders: perspectives from humans and rodent models. Biology of Sex Differences 5.

Shukla, R., Oh, H., Sibille, E., 2020. Molecular and Cellular Evidence for Age by Disease Interactions: Updates and Path Forward. The American Journal of geriatric psychiatry 28, 237247.

Son, H., Banasr, M., Choi, M., Chae, S. Y., Licznerski, P., Lee, B., Voleti, B., Li, N., Lepack, A., Fournier, N. M., 2012. Neuritin produces antidepressant actions and blocks the neuronal and behavioral deficits caused by chronic stress. Proceedings of the National Academy of Sciences 109, 11378-11383.

Steadman, P. E., Ellegood, J., Szulc, K. U., Turnbull, D. H., Joyner, A. L., Henkelman, R. M., Lerch, J. P., 2014. Genetic effects on cerebellar structure across mouse models of autism using a magnetic resonance imaging atlas. Autism research 7, 124-137.

Steffens, D. C., McQuoid, D. R., Payne, M. E., Potter, G. G., 2011. Change in hippocampal volume on magnetic resonance imaging and cognitive decline among older depressed and nondepressed subjects in the neurocognitive outcomes of depression in the elderly study. The American Journal of geriatric psychiatry 19, 4-12.

Stockmeier, C. A., Mahajan, G. J., Konick, L. C., Overholser, J. C., Jurjus, G. J., Meltzer, H. Y., Uylings, H. B. M., Friedman, L., Rajkowska, G., 2004. Cellular changes in the postmortem hippocampus in major depression. Biological Psychiatry 56, 640-650.

Tang, Y. Y., Kong, L., Wu, F., Womer, F., Jiang, W., Cao, Y., Ren, L., Wang, J., Fan, G., Blumberg, H. P., Xu, K., Wang, F., 2013. Decreased functional connectivity between the amygdala and the left ventral prefrontal cortex in treatment-naive patients with major depressive disorder: a resting-state functional magnetic resonance imaging study. Psychol Med 43, 19211927.

Treadway, M. T., Waskom, M. L., Dillon, D. G., Holmes, A. J., Park, M. T. M., Chakravarty, M. M., Dutra, S. J., Polli, F. E., Iosifescu, D. V., Fava, M., Gabrieli, J. D. E., Pizzagalli, D. A., 2015. 
Illness Progression, Recent Stress, and Morphometry of Hippocampal Subfields and Medial Prefrontal Cortex in Major Depression. Biological Psychiatry 77, 285-294.

Uezato, A., Meador-Woodruff, J. H., McCullumsmith, R. E., 2009. Vesicular glutamate transporter mRNA expression in the medial temporal lobe in major depressive disorder, bipolar disorder, and schizophrenia 11, 711-725.

Ullmann, J. F., Watson, C., Janke, A. L., Kurniawan, N. D., Reutens, D. C., 2013. A segmentation protocol and MRI atlas of the C57BL/6J mouse neocortex. Neuroimage 78, 196203.

Van Tol, M.-J., Van Der Wee, N. J. A., Van Den Heuvel, O. A., Nielen, M. M. A., Demenescu, L. R., Aleman, A., Renken, R., Van Buchem, M. A., Zitman, F. G., Veltman, D. J., 2010. Regional Brain Volume in Depression and Anxiety Disorders. Archives of General Psychiatry 67, 1002.

Videbech, P., 2004. Hippocampal Volume and Depression: A Meta-Analysis of MRI Studies. American Journal of Psychiatry 161, 1957-1966.

Vyas, A., Jadhav, S., Chattarji, S., 2006. Prolonged behavioral stress enhances synaptic connectivity in the basolateral amygdala. Neuroscience 143, 387-393.

Vyas, A., Mitra, R., Rao, B. S., Chattarji, S., 2002. Chronic stress induces contrasting patterns of dendritic remodeling in hippocampal and amygdaloid neurons. Journal of Neuroscience 22, 6810-6818.

Webb, C., Weber, M., Mundy, E., Killgore, W., Reduced gray matter volume in the anterior cingulate, orbitofrontal cortex and thalamus as a function of mild depressive symptoms: a voxelbased morphometric analysis. Psychol Med 44, 2833-2843.

Wellman, C. L., 2001. Dendritic reorganization in pyramidal neurons in medial prefrontal cortex after chronic corticosterone administration. Journal of neurobiology 49, 245-253.

Wheeler, A. L., Wessa, M., Szeszko, P. R., Foussias, G., Chakravarty, M. M., Lerch, J. P., DeRosse, P., Remington, G., Mulsant, B. H., Linke, J., Malhotra, A. K., Voineskos, A. N., 2015. Further neuroimaging evidence for the deficit subtype of schizophrenia: a cortical connectomics analysis. JAMA Psychiatry 72, 446-455.

Willner, P., 2017. The chronic mild stress (CMS) model of depression: History, evaluation and usage. Neurobiology of Stress 6, 78-93.

Ye, M., Yang, T., Qing, P., Lei, X., Qiu, J., Liu, G., 2015. Changes of Functional Brain Networks in Major Depressive Disorder: A Graph Theoretical Analysis of Resting-State fMRI. PLoS ONE 10, e0133775.

Yee, Y., Fernandes, D. J., French, L., Ellegood, J., Cahill, L. S., Vousden, D. A., Spencer Noakes, L., Scholz, J., van Eede, M. C., Nieman, B. J., Sled, J. G., Lerch, J. P., 2018. Structural covariance of brain region volumes is associated with both structural connectivity and transcriptomic similarity. Neuroimage 179, 357-372.

Yu, H., Li, M., Zhou, D., Lv, D., Liao, Q., Lou, Z., Shen, M., Wang, Z., Li, M., Xiao, X., Zhang, Y., Wang, C., 2018. Vesicular glutamate transporter 1 (VGLUT1)-mediated glutamate release and membrane GluA1 activation is involved in the rapid antidepressant-like effects of scopolamine in mice. Neuropharmacology 131, 209-222.

Zhao, Y., Du, M., Huang, X., Lui, S., Chen, Z., Liu, J., Luo, Y., Wang, X., Kemp, G., Gong, Q., 2014. Brain grey matter abnormalities in medication-free patients with major depressive disorder: a meta-analysis. Psychol Med 44, 2927-2937.

Zhuo, C., Li, G., Lin, X., Jiang, D., Xu, Y., Tian, H., Wang, W., Song, X., 2019. The rise and fall of MRI studies in major depressive disorder. Translational Psychiatry 9. 
Zink, M., Vollmayr, B., Gebicke-Haerter, P. J., Henn, F. A., 2010. Reduced expression of glutamate transporters vGluT1, EAAT2 and EAAT4 in learned helpless rats, an animal model of depression. Neuropharmacology 58, 465-473. 


\section{Figure Legends}

Figure 1: Chronic restraint stress exposure induces deficits in behavioral emotionality and working memory. (A) Schematic representation of the experimental design. Control animals and mice subjected to 2 or 5 weeks of chronic restraint stress (CRS) (n=12/group; 50\% males) were tested in series of behavioral tests throughout the experiment (gray) and during the $5^{\text {th }}$ week of the experiment (black). CRS-exposed mice showed increased latency to bite in the (B) novelty suppressed feeding test (NSF) and increased residual avoidance in the (C) PhenoTyper test (PT). CRS animals also showed reduced sucrose consumption in the (D) sucrose consumption test (SC). While coat state degradation (E) was found significantly elevated in CRS animals. Overall CRS induced an increase in z-emotionality (F) calculated from the mean z-scores of the novelty suppressed feeding, PhenoTyper and sucrose consumption tests. CRS mice also displayed deficits in the $(\mathrm{G})$ Y-maze working memory test. ${ }^{*} \mathrm{p}<0.05$ compared to controls and ${ }^{\alpha} \mathrm{p}<0.05$ compared to 2 weeks CRS. Individual males $(\Delta)$ and females $(\mathrm{O})$ are represented in each figure.

Figure 2: Chronic stress-induced deficits in behavioral emotionality and total brain volume changes are greater in female mice. Male and female mice were exposed or not to chronic restraint stress (CRS) (n=6/group). (A) Overall z-emotionality scores were calculated for each group and each sex. We found a decreased in (B) total brain volume (TBV) in female mice exposed to 2 and 5 weeks of CRS. Scatter plot showing the correlation (95\% confidence interval) between (C) TBV changes and z-emotionality. A significant negative correlation was found when all animals ( $\mathrm{r}=-.49 ; \mathrm{P}=0.002$; black line), or only females ( $\mathrm{r}=-0.59 ; \mathrm{P}=0.01$; dotted grey line) were considered, but not when only males (grey line) considered. Individual males $(\triangle)$ and females $(\mathrm{O})$ are represented in each figure. ${ }^{*} \mathrm{p}<0.05$ as compared to respective controls; ${ }^{\beta} \mathrm{p}<0.05$ as compared to males.

Figure 3: Chronic stress effects on 4 corticolimbic brain region volumes and correlation between regional volume and behavioral emotionality. Chronic restraint stress (CRS) induced a decreased in absolute volume of the (A) anterior cingulate cortex $\left({ }^{*} \mathrm{p}<0.05\right.$ as compared to controls). No significant changes in absolute volume were found in the (C) hippocampus, (E) amygdala and $(G)$ nucleus accumbens. Scatter plots display correlations $(95 \%$ confidence interval) between z-emotionality score and volume changes in the (B) anterior cingulate cortex 
$(\mathrm{r}=-0.56 ; \mathrm{p}=0.0003),(\mathrm{F})$ amygdala $(\mathrm{r}=-0.35 ; \mathrm{p}=0.03)$, and $(\mathrm{H})$ nucleus accumbens $(\mathrm{r}=-0.41 ; \mathrm{p}=$ 0.01). No significant correlation observed in the (D) hippocampus. Individual males $(\Delta)$ and females $(\mathrm{O})$ are represented in each figure.

Figure 4: Chronic stress induced progressive decrease in strength and degree of the anterior cingulate cortex (ACC) and increase in strength and degree of the amygdala structural covariance networks. Decrease in ACC degree (A) were found in mice exposed to 2 and 5 weeks of chronic restraint stress (CRS), relative to mice exposed to no stress (controls). Permutation testing revealed significant differences in degree when comparing the 5 weeks CRS group to the control group at density $=0.13-0.19$ (yellow) $(\mathrm{p}<.05$ dashed, $\mathrm{p}<.10$ (dotted)) Subnetwork visualization $(B)$ at density $=0.13$ was performed using the Cytoscape EdgeWeighted Spring Embedded Layout. Increase in amygdala degree (C) were found in mice exposed to 2 and 5 weeks chronic restraint stress (CRS), relative to mice exposed to no stress (controls). Permutation testing revealed significant differences in degree when comparing the 5week CRS group to the control group at density $=0.08-0.12$ (yellow) $(\mathrm{p}<.05$ dashed, $\mathrm{p}<.10$ (dotted)). Subnetwork visualization (D) at density $=0.08$ was used. Nodes labeled in red are common to the no stress and 2 weeks CRS groups; nodes labeled in yellow are common to the no stress and 5 weeks CRS groups; nodes labeled in green are common to all three stress groups; and nodes labeled in blue are unique to each stress group. Node numbers represent brain regions listed in Supplementary Table 6 (ACC) and 7 (Amygdala). The length of each edge represents the strength of correlations between nodes.

Figure 5: Changes in PSD95 puncta density are linked to volume of the anterior cingulate cortex (ACC) and behavioral emotionality. Max projection of three-dimension (3D) image zstacks of top $\sim 4 \mu \mathrm{m}$ of tissue imaged at $0.1 \mu \mathrm{m} / \mathrm{stack}$ of (A) original confocal image of PSD95 immunolabelling and (B) blind deconvoluted image. (C) Image quantification performed using IMARIS software identifies quality of detected puncta based on intensity at center of each spot in 3D. CRS did not induce any significant changes in PSD95 puncta density of mouse anterior cingulate cortex (D). Scatter plots display a significant correlation (95\% confidence interval) of changes in PSD95 puncta density with (E) ACC volume ( $\mathrm{r}=0.35 ; \mathrm{p}=0.044)$, and $(\mathrm{F})$ behavioral emotionality $(\mathrm{r}=-0.439 ; \mathrm{p}=0.009)$. Individual males $(\Delta)$ and females $(\mathrm{O})$ are represented in each figure. 
Figure 1

A

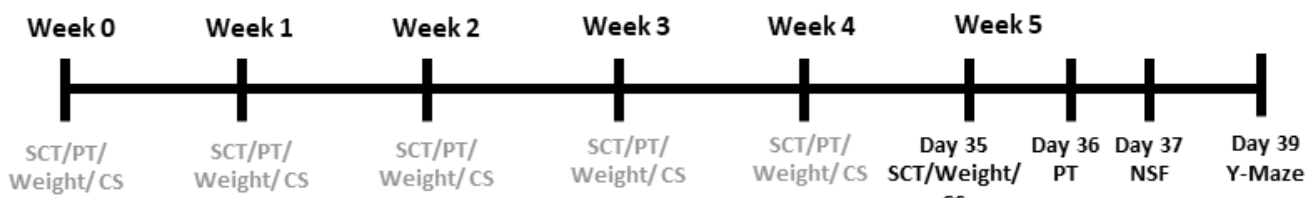

CS

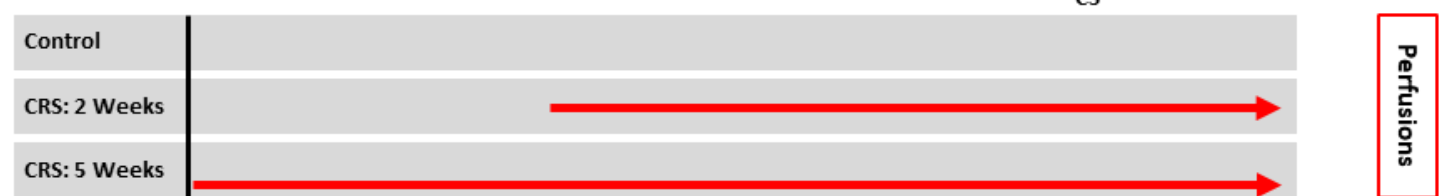

Novelty Suppressed feeding (NSF)

Residual Avoidance (RA)

Sucrose Consumption

B

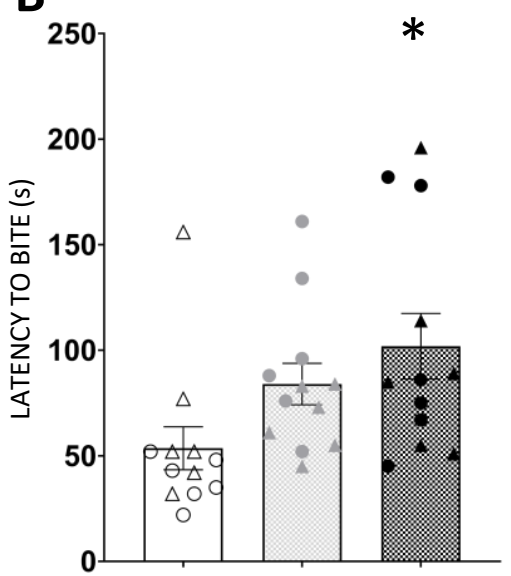

No Stress 2 Weeks 5 Weeks CRS CRS
C

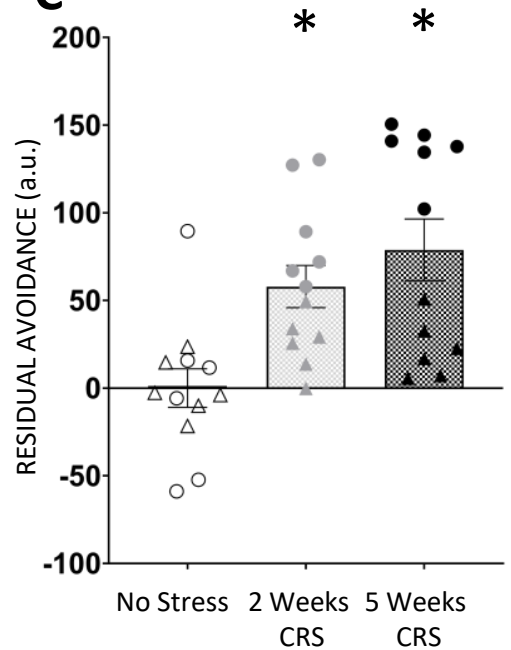

D

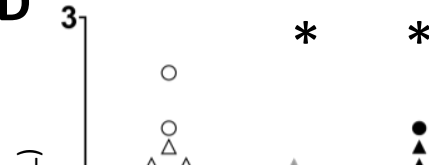

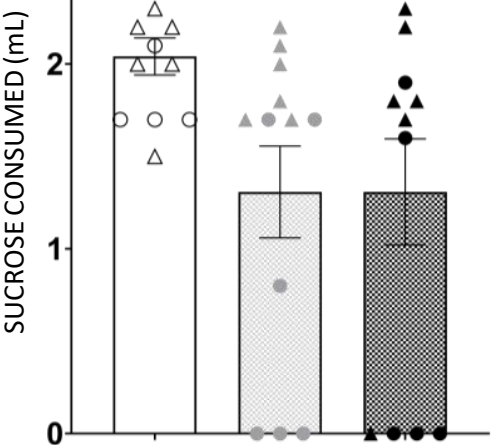

No Stress 2 Weeks 5 Weeks CRS CRS

E

Coat State Quality

$\mathbf{F}$

Z-Emotionality

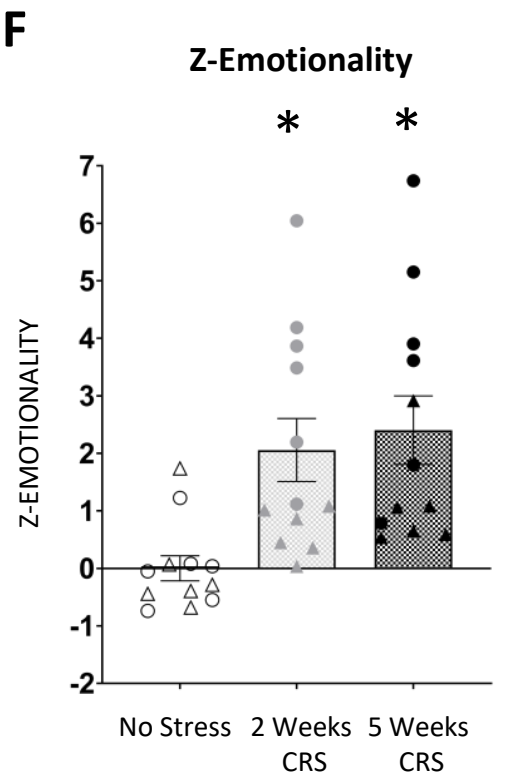

$\mathbf{G}$

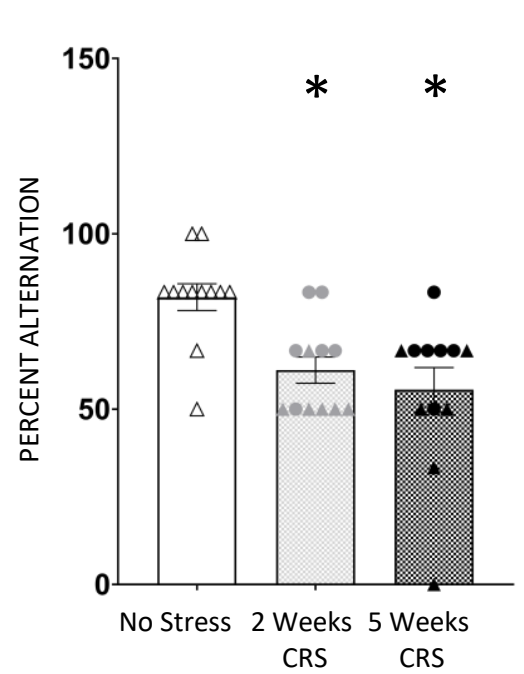

Y-Maze 
Figure 2
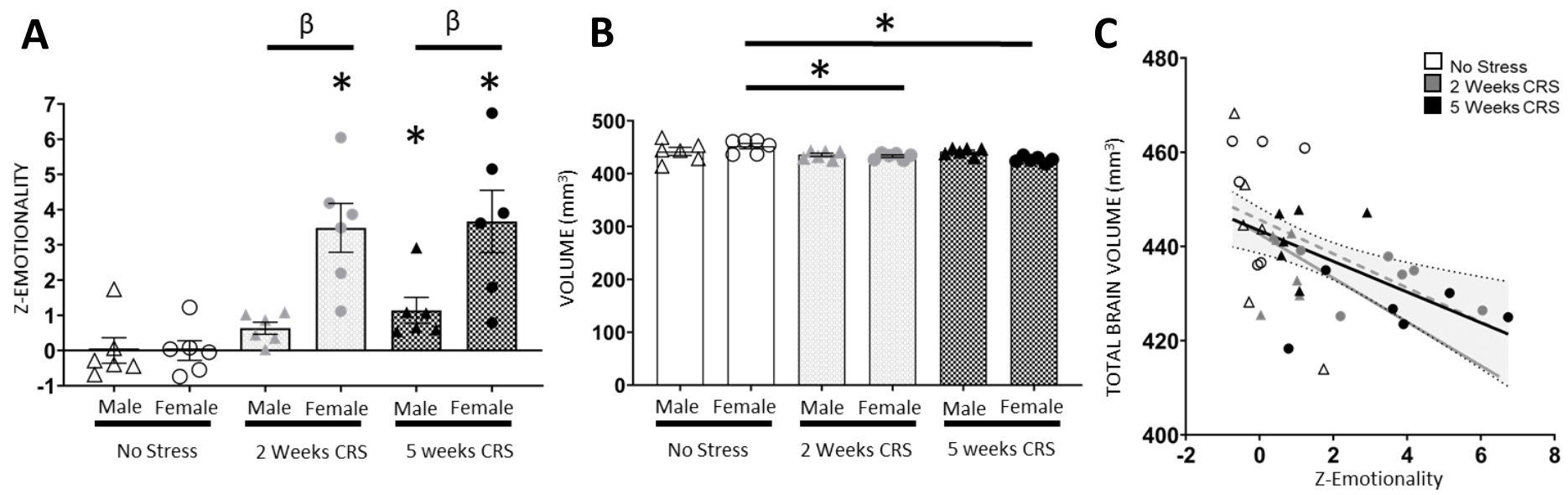


\section{Figure 3}

\section{Anterior Cingulate Cortex}

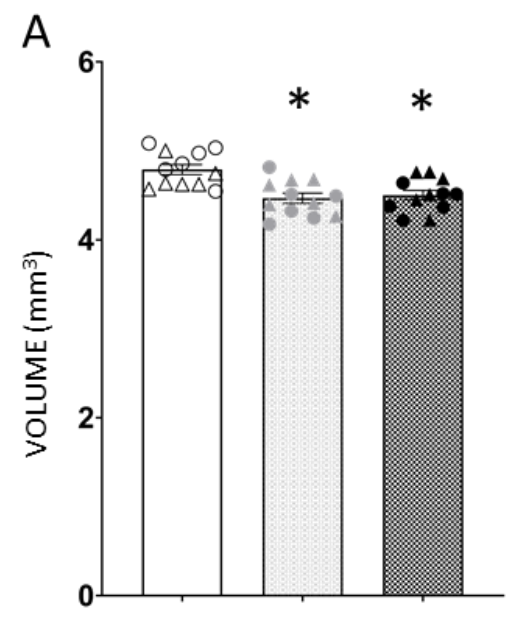

No Stress 2 Weeks 5 Weeks

CRS

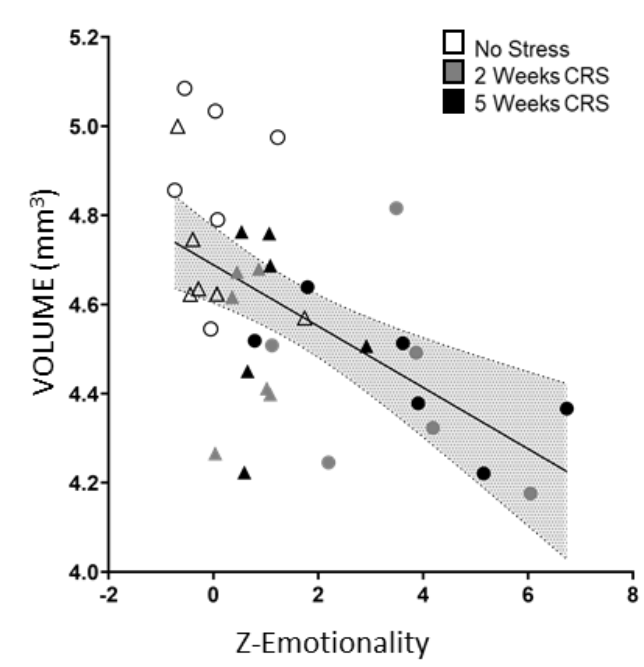

Amygdala

E

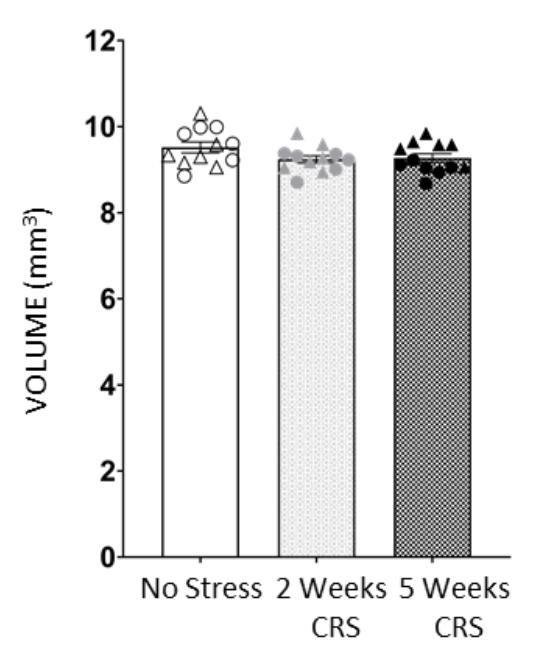

$\mathrm{F}$

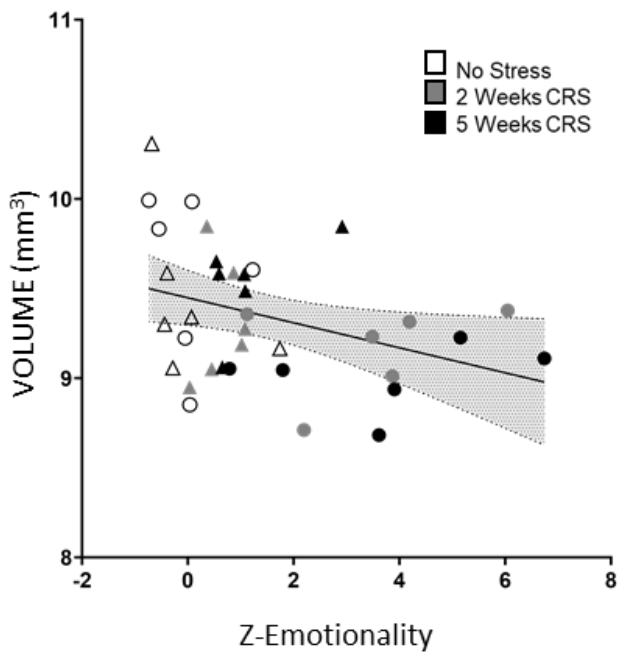

\section{Hippocampus}

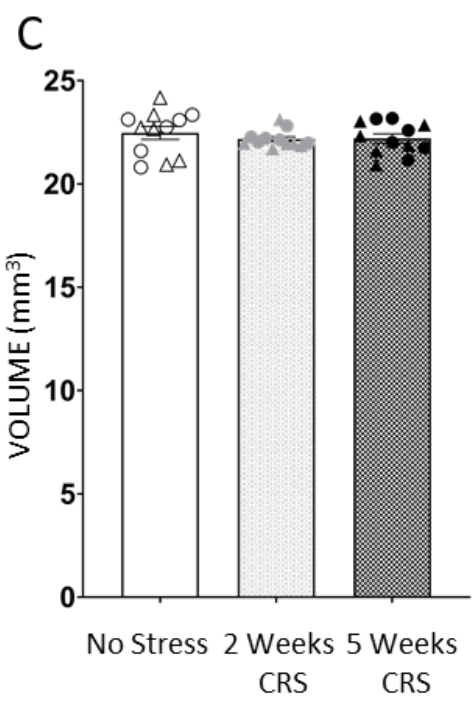

D

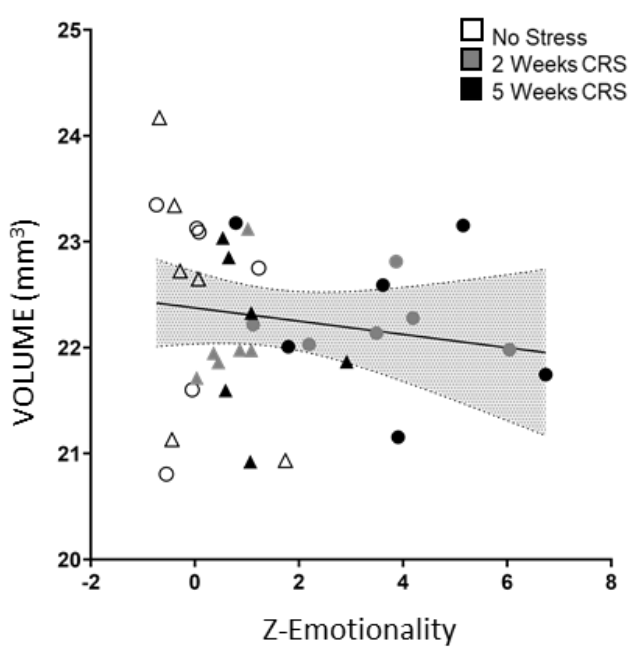

\section{Nucleus Accumbens}

G

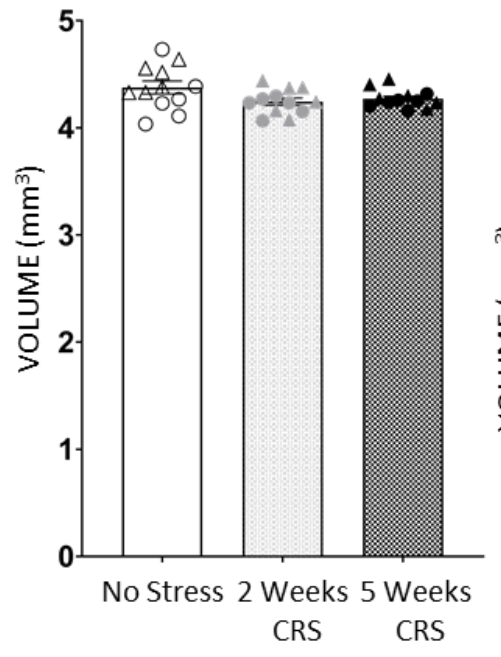

$\mathrm{H}$

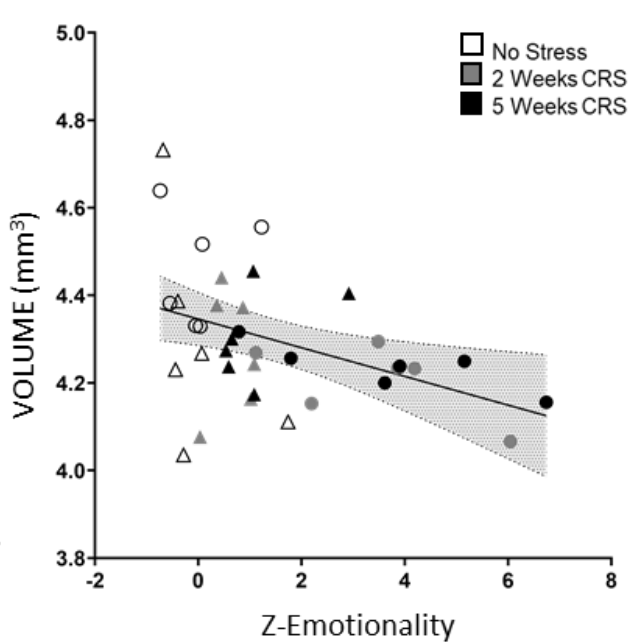




\section{Figure 3}

\section{Anterior Cingulate Cortex}

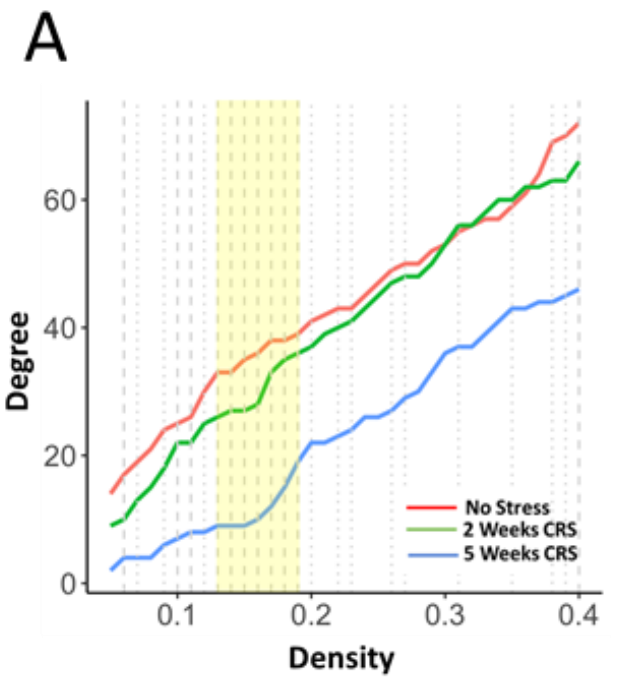

B

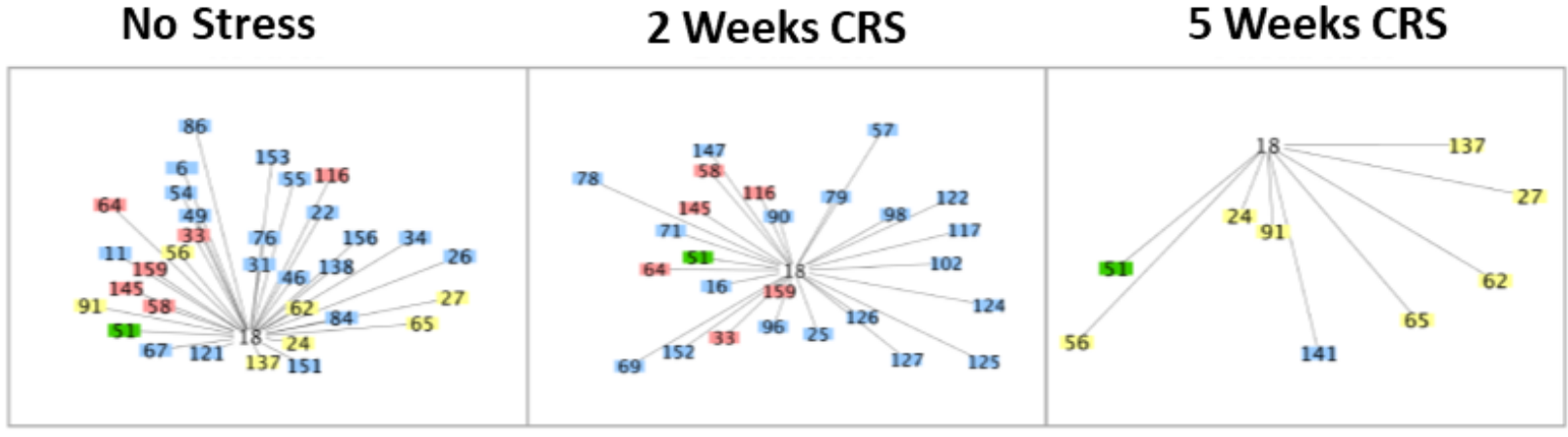

O Group-specific O No Stress +2 weeks CRS O No Stress +5 weeks CRS O All Groups

\section{Amygdala}

C

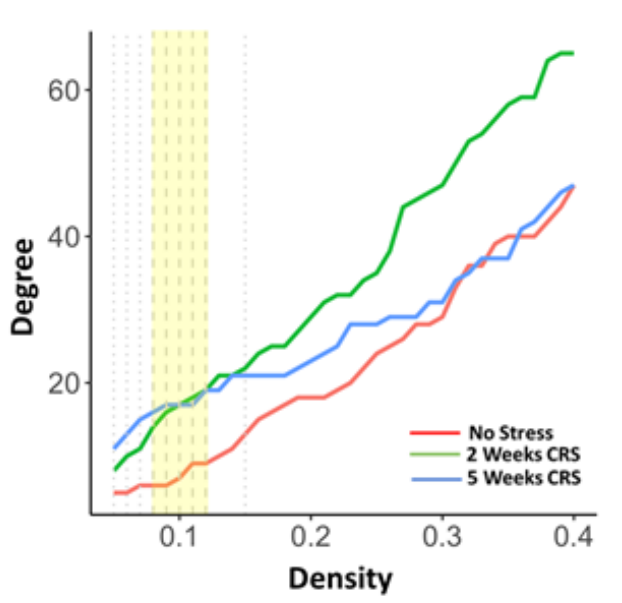

D

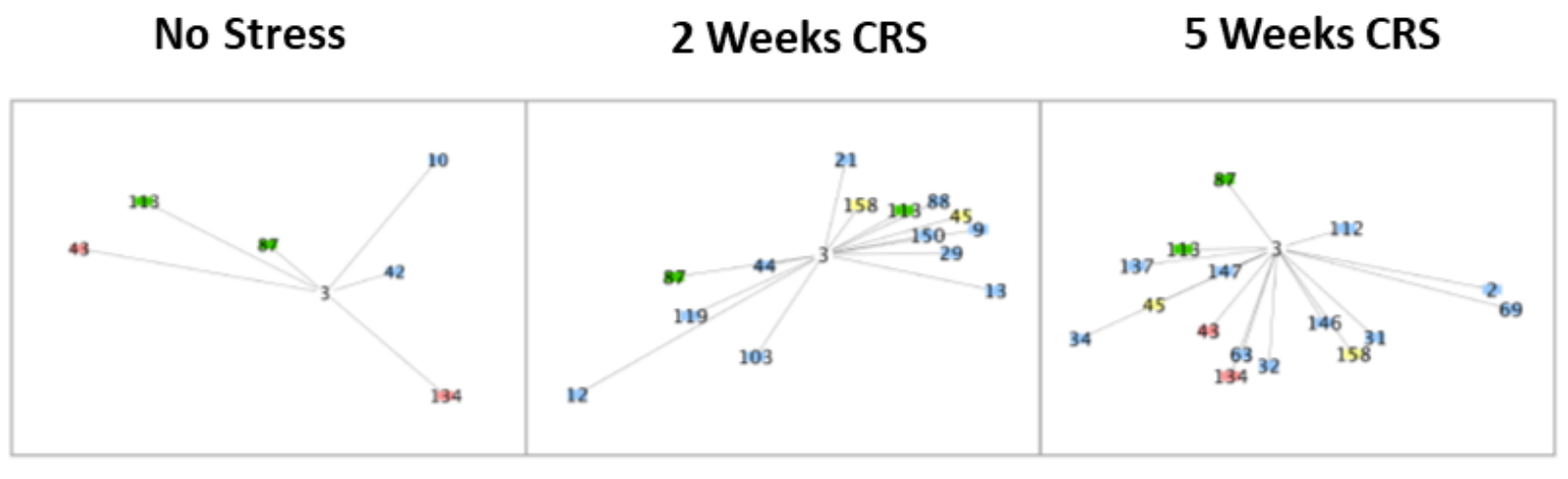

O Group-specific O No Stress+ 2 weeks CRS O No Stress+ 5 weeks CRS $\circ$ All Groups 


\section{Figure 5}
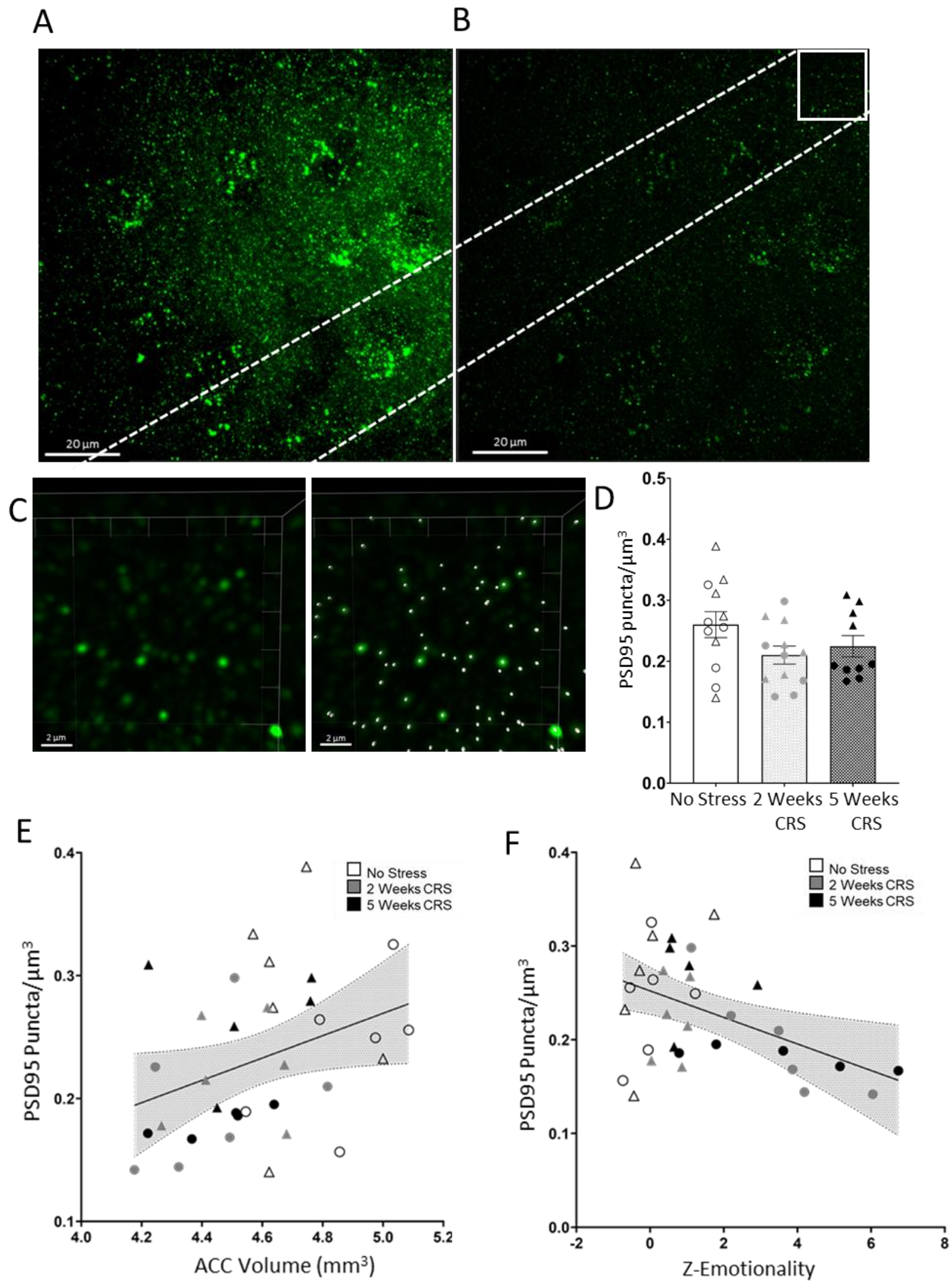